\title{
Utilizing Bioinformatics Technology to Explore the Potential Mechanism of Danggui Buxue Decoction against NSCLC
}

\author{
Bin Yu $\mathbb{D}^{1},{ }^{1}$ Guangyao Lv ${ }^{D},{ }^{1}$ Muhammad Sohail $\mathbb{D}^{1},{ }^{1}$ Zhiyong Li ${ }^{\mathbb{D}},{ }^{1,2}$ Yanli Li $\left(\mathbb{D},{ }^{1}\right.$ \\ Meiyu Yu $\mathbb{D}^{3},{ }^{3}$ Fuyou Sun $\mathbb{D}^{3},{ }^{3}$ and Hui Xu $\mathbb{D}^{1}$ \\ ${ }^{1}$ School of Pharmacy, Collaborative Innovation Center of Advanced Drug Delivery System and Biotech Drugs in Universities \\ of Shandong, Key Laboratory of Molecular Pharmacology and Drug Evaluation, Yantai University, Yantai, \\ Shandong Province, China \\ ${ }^{2}$ Guangdong Zhongrun Pharmaceutical R\&D Co., Ltd., Guangzhou, Guangdong Province, China \\ ${ }^{3}$ Department of Pharmaceutics, Yantai Muping Hospital of Traditional Chinese Medicine, Yantai, Shandong Province, China
}

Correspondence should be addressed to Hui Xu; xuhui@ytu.edu.cn

Received 5 December 2021; Accepted 10 February 2022; Published 26 February 2022

Academic Editor: Andy T. Y. Lau

Copyright (c) 2022 Bin Yu et al. This is an open access article distributed under the Creative Commons Attribution License, which permits unrestricted use, distribution, and reproduction in any medium, provided the original work is properly cited.

\begin{abstract}
While lung cancer poses a serious threat to human health, non-small-cell lung cancer (NSCLC) is the most common type of lung cancer. Danggui Buxue Decoction (DBD) is a classical traditional antitumor medicine commonly used in China. However, the potential mechanism of DBD against NSCLC has not yet been expounded. Therefore, this study clarified the potential molecular mechanism and key targets of DBD in NSCLC treatment through several technological advances, such as network pharmacology, molecular docking, and bioinformatics. Firstly, the relative active ingredients and key DBD targets were analyzed, and subsequently, a drug-ingredient-target-disease network diagram was constructed for NSCLC treatment with $\mathrm{DBD}$, resulting in the identification of five main active ingredients and ten core targets according to the enrichment degree. The enrichment analysis revealed that DBD can achieve the purpose of treating NSCLC through the AGE-RAGE signaling pathway in diabetic complications. Secondly, the molecular docking approach predicted that quercetin and hederagenin have the best working mechanisms with PDE3A and PTGS1, while the survival analysis results depicted that high PDE3A gene expression has a relatively poor prognosis for NSCLC patients $(p<0.05)$. Additionally, PDE3A is mainly distributed in the LU65 cell line that originated from Asian population. In summary, our study results showed that DBD can treat NSCLC through the synergistic correlation between multiple ingredients, multiple targets, and multiple pathways, thus effectively improving NSCLC prognosis. This study not only reflected the medicinal value of DBD but also provided a solid structural basis for future new drug developments and targeted therapies.
\end{abstract}

\section{Introduction}

According to the estimates from World Health Organization's "Global Cancer Report 2020," lung cancer (2.21 million cases) remains the second most commonly diagnosed cancer in the world [1]. The report also predicts that by 2040 , due to the incidence rate being $20 \%$, the number of new cancer cases will exceed 27 million worldwide, with one in every five people suffering from cancer. In 2020, an estimated 4.57 million new cancer cases were reported in China, of which lung cancer is by far the leading cause of the highest number of reported cases at $23.7 \%$ and is still the most frequently occurring cancer threatening the health of the Chinese people [1-3]. Among all the lung cancer subtypes, NSCLC is the most common form, accounting for $80 \%$ of all lung cancer diagnoses with a 5 -year survival rate of $18 \%[4,5]$. As the NSCLC mortality rate is reaching towering proportions every year [6], the conventional treatment regime is still based on chemotherapy or radiotherapy, which predicts a poorer prognosis, reduces the quality of life, and increases the economic burden on patients and their families through expensive treatment costs [7]. Although the emergence of targeted therapy is now being considered a boon for cancer patients, the resultant increase in drug resistance and unexplained incidence of allergic reactions 
make it inappropriate for some patients $[8,9]$. In the present scenario, several other studies involved in research on traditional Chinese medicine (TCM) have also postulated that TCM has its unique advantages while treating tumors with significant curative effects, fewer side effects, and low drug resistance and can also manifest excellent outcomes in combination with other conventional drugs [10-13].

As described in Li Gao's "Differentiation of Endogenous and Exogenous Disorders," DBD is a fixed-ratio $(5: 1)$ coformulation consisting of Chinese medicines Danggui and Huangqi, which is also considered as a classic formula for invigorating qi and producing blood, thus increasing hematopoietic function [14]. Danggui is the dried root of a perennial leguminous plant Astragalus membranaceus (Fisch.) Bge. var. mongholicus (Bge.) Hsiao (AMB) [15] whereas Huangqi is the dry root of Angelica sinensis (Oliv.) Diels (family Umbellaceae) [16]. A plethora of pharmacological studies revealed that $\mathrm{DBD}$ regulates immune responses [17], promotes hematopoiesis [17, 18], protects the cardiovascular system [19], shields the liver [20], and demonstrates antitumor properties [21]. DBD has a good clinical effect on NSCLC and can effectively improve the quality of life of patients with advanced NSCLC; when used alone or in combination with other conventional drugs, it can also reduce the side effects of radiotherapy and chemotherapy as well as improve their sensitivity [22-25]. Although it was observed in clinical practice that DBD is highly effective in the NSCLC treatment, the complex composition of DBD and the unclear mechanism of action make it quite difficult to conduct further research. Henceforth, TCM being a traditional medicine can also treat diseases through multicomponent, multitarget, and multipathway synergistic effects to achieve the purpose of curative healing [26]. However, the interconnected complexity of traditional Chinese medicine components and their indistinct mechanism of action duly limit further development and usage of such traditional medicines [27].

As a novel research approach, network pharmacology is based on high-throughput omics data analysis, computer virtual computing, and network database retrieval, which acts as an effective means for discovering active drug substances and revealing their pharmacological mechanisms of action from a crucial perspective of molecular biological networks [28]. The multicomponent, multitarget, and multipathway characteristics of Chinese herbal compound prescriptions are the prime focus domain of network pharmacology research [29]. Although molecular docking is mainly used to study the interaction between the molecules and predict salient binding modes and relationships, it is also a mathematical simulation of drug-receptor interactions to predict the most active drug component [30].

Owing to the fact that there is no network pharmacology study in the literature to date on NSCLC treatment with DBD, this study explored the functional molecular mechanism of DBD in NSCLC treatment through network pharmacology and molecular docking as well as analyzed the survival of important targets through The Cancer Genome Atlas (TCGA) database to duly assess the target's impact on NSCLC prognosis. In addition, this study also conducted an in-depth analysis of the expression of important targets in NSCLC tumor cell lines through the Cancer Cell Line Encyclopedia (CCLE) database. Our research plan is shown in Figure 1.

\section{Materials and Methods}

2.1. Collection of Active Ingredients and Chemical Structure of DBD. Traditional Chinese medicine systems pharmacology (TCMSP) database (https://tcmsp-e.com/) was used to collect the active ingredients of DBD. For identifying the active ingredients in DSD, "Danggui" was heavily explored in the database, followed by scheduling screening conditions through oral bioavailability (OB) and druglikeness (DL) parameters to acquire the needed active ingredients and downloading them. Since OB represents the percentage of traditional Chinese medicine's absorption in the human circulatory system, while DL is the similarity between the ingredients and known drugs [31], the following values $(\mathrm{OB}>30 \%$ and $\mathrm{DL}>0.18)$ were decided as the foremost screening conditions of all active ingredients. "Huangqi" was also profoundly searched through the TCMSP database and was filtered and downloaded according to these values $(\mathrm{OB}>30 \%$ and $\mathrm{DL}>0.18)$ set as the screening conditions.

The TCMSP database contains the chemical structure of all the active ingredients. The corresponding chemical structure of obtained active ingredients can also be downloaded through the TCMSP database. Additionally, active ingredients' Tripos molecule structure format (MOL2) files were also obtained for further utilization in the molecular docking procedure. Due to the nonavailability of information about the main active ingredients, MOL2 files of all active ingredients were downloaded for further screening processes. However, in the case of active ingredients lacking MOL2 files, ChemBioDraw Ultra and ChemBio3D Ultra software were used to draw them and later saved them in MOL2 format for further usage.

2.2. The Potential Target Database of NSCLC. We used the GeneCards database (https://www.genecards.org/) and Online Mendelian Inheritance in Man (OMIM database) (https://omim.org/) to search the NSCLC-related targets. For instance, after inserting "Non-Small Cell Lung Cancer" as a keyword in two databases, the relevant targets downloaded in databases were merged and then deduplicated to acquire the NSCLC relevant targets. After the utilization of R software to draw a Venn diagram of DBD and NSCLC targets, finally, our core targets were achieved.

2.3. Protein-Protein Interaction Construction. The construction of the protein-protein interaction (PPI) network was completed on the string (https://string-db.org/) website. Firstly, the "Multiple Proteins" option was selected, followed by mentioning the core targets in the List of Names and inserting "homo sapiens" in the Organism inquiry. Lastly, after inserting the default values for other parameters, the PPI network map was obtained for our perusal.

2.4. Drug-Ingredient-Target-Disease Network Construction. A Cytoscape software was employed to build a drug- 


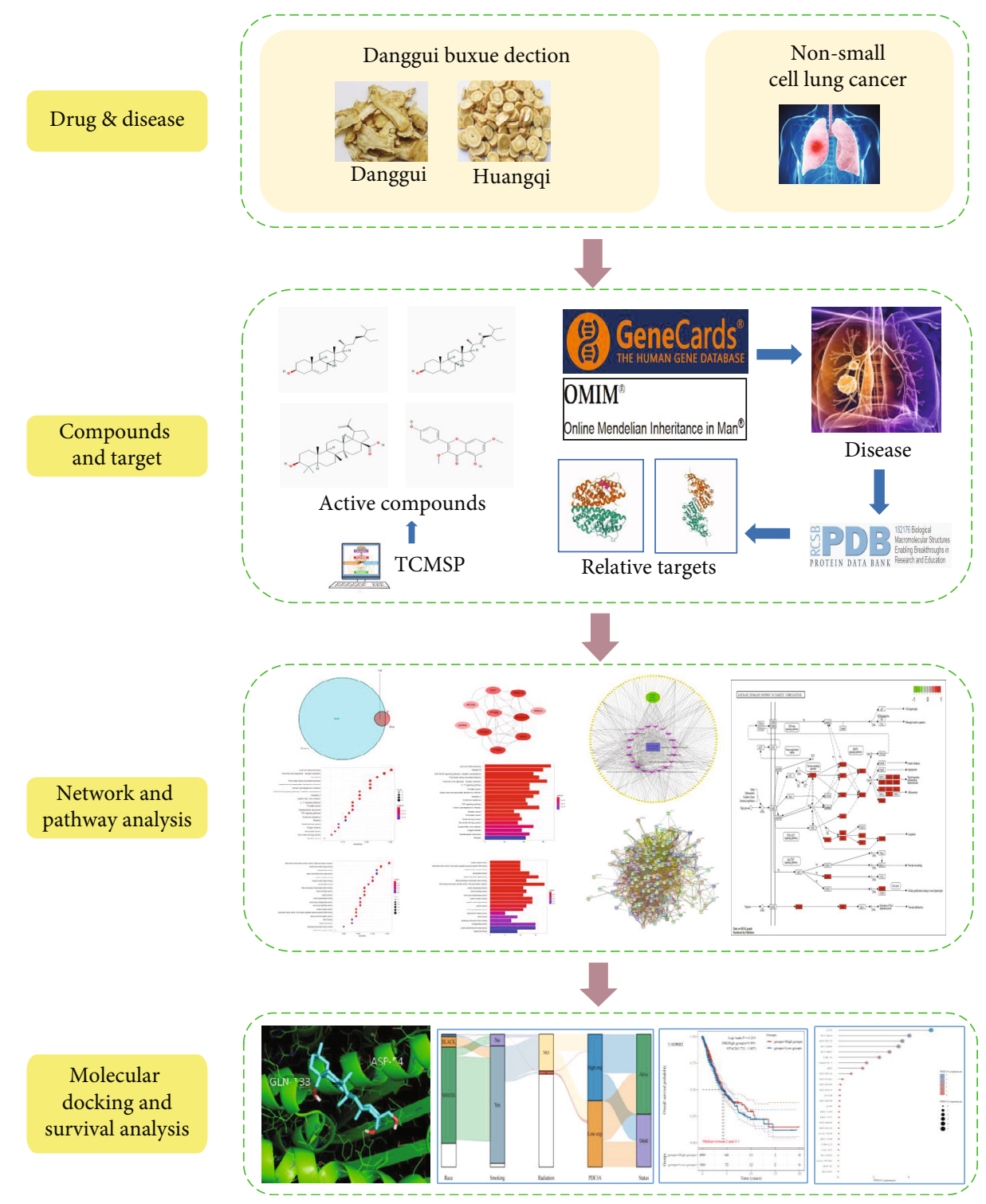

Figure 1: Technological plan for this study.

ingredient-target-disease visualization network diagram. After inserting the obtained drugs, ingredients, core targets, diseases, and their mutual correspondence mechanisms into the Cytoscape software, the topological characteristics of network nodes were calculated through the Cytoscape plugin Network Analyzer that included various degrees of correlation, namely, degree, betweenness, and closeness, among which the degree value was an important evaluation index [32]. Subsequently, ten main targets were also acquired through the degree correlation factor of the Cytoscape software.

2.5. GO Functional Analysis and KEGG Enrichment Pathway Analysis. In order to further understand the function of the core targets and the key DBD pathway against NSCLC, a Gene Ontology (GO) functional enrichment analysis and Kyoto Encyclopedia of Genes and Genomes (KEGG) enrichment pathway analysis were conducted. GO functional enrichment analysis was performed by using Cytoscape software and $\mathrm{R}$ software while the results consisted of an amalgamation of biological process, cellular component, and molecular function and were presented in a bubble chart and a histogram with a $p$ value where $p<0.05$ indicated statistical significance. KEGG enrichment pathway analysis was also performed through Cytoscape software and R software, while their results included a bubble chart, a histogram, and some signal path diagrams with a $p$ value; $p<0.05$ indicated statistical significance.

2.6. Molecular Docking. AutoDock Vina software was used to verify the molecular docking mechanism between the top ten key targets in the PPI network and the five main active DBD ingredients. The three-dimensional structure of the ten target proteins from the RCSB PDB protein structure database (https://www.rcsb.org/) and the chemical structure of Tripos molecule structure format (MOL2) file of active 
TABLE 1: Information of active ingredients and related targets from DBD.

\begin{tabular}{|c|c|c|c|c|c|c|}
\hline $\begin{array}{l}\text { Herb } \\
\text { name }\end{array}$ & Mol ID & Mol name & Mol structure & Related targets & OB (\%) & $\mathrm{DL}$ \\
\hline \multirow[t]{2}{*}{ Danggui } & MOL000358 & Beta-sitosterol & & $\begin{array}{c}\text { PGR, NCOA2, PTGS1, PTGS2, } \\
\text { HSP90AA1, KCNH2, PRKACA, } \\
\text { DRD1, CHRM3, CHRM1, SCN5A, } \\
\text { CHRM4, PDE3A, ADRA1A, } \\
\text { CHRM2, ADRA1B, ADRB2, } \\
\text { CHRNA2, SLC6A4, OPRM1, } \\
\text { CHRNA7, BCL2, BAX, CASP9, } \\
\text { JUN, CASP3, CASP8, PRKCA, } \\
\text { PON1, MAP2 }\end{array}$ & 36.91 & 0.75 \\
\hline & MOL000449 & Stigmasterol & & $\begin{array}{c}\text { PGR, NR3C2, NCOA2, RXRA, } \\
\text { NCOA1, PTGS1, PTGS2, ADRA2A, } \\
\text { SLC6A2, SLC6A3, ADRB2, } \\
\text { AKR1B1, PLAU, LTA4H, MAOB, } \\
\text { MAOA, PRKACA, CTRB1, } \\
\text { CHRM3, CHRM1, ADRB1, SCN5A, } \\
\text { ADRA1A, CHRM2, ADRA1B, } \\
\text { CHRNA7 }\end{array}$ & 43.83 & 0.75 \\
\hline & MOL000211 & Mairin & & PGR & 55.38 & 0.78 \\
\hline & MOL000239 & Jaranol & & $\begin{array}{c}\text { NOS2, PTGS1, AR, SCN5A, PTGS2, } \\
\text { ESR2, DPP4, HSP90AA1, CHEK1, } \\
\text { PRSS1, NCOA2 }\end{array}$ & 50.83 & 0.29 \\
\hline & MOL000296 & Hederagenin & & $\begin{array}{c}\text { PGR, NCOA2, CHRM3, CHRM1, } \\
\text { CHRM2, ADRA1B, GRIA2, LYZ, } \\
\text { PTGS1, SCN5A, PTGS2, RXRA, } \\
\text { PDE3A, SLC6A2 }\end{array}$ & 36.91 & 0.75 \\
\hline Huangqi & MOL000033 & $\begin{array}{c}\text { (3S,8S,9S,10R,13R,14S,17R)- } \\
\text { 10,13-Dimethyl-17-[(2R,5S)-5- } \\
\text { propan-2-yloctan-2-yl]- } \\
\text { 2,3,4,7,8,9,11,12,14,15,16,17- } \\
\text { dodecahydro-1H- } \\
\text { cyclopenta[a]phenanthren-3-ol }\end{array}$ & & PGR & 36.23 & 0.78 \\
\hline & MOL000354 & Isorhamnetin & & $\begin{array}{l}\text { NOS2, PTGS1, ESR1, AR, PPARG, } \\
\text { PTGS2, PTPN1, ESR2, DPP4, } \\
\text { MAPK14, GSK3B, HSP90AA1, } \\
\text { PRKACA, PRSS1, CCNA2, NCOA2, } \\
\text { PYGM, PPARD, CHEK1, AKR1B1, } \\
\text { NCOA1, F7, F2, ACHE, MAOB, } \\
\text { GRIA2, RELA, NCF1, OLR1 }\end{array}$ & 49.60 & 0.31 \\
\hline & MOL000371 & 3,9-Di-O-methylnissolin & & $\begin{array}{c}\text { NOS2, PTGS1, CHRM3, F2, } \\
\text { CHRM1, ESR1, ADRB1, SCN5A, } \\
\text { PTGS2, ADRA2C, RXRA, ACHE, } \\
\text { PDE3A, ADRA1B, ADRB2, } \\
\text { ADRA1D, OPRM1, PRSS1, NCOA2 }\end{array}$ & 53.74 & 0.48 \\
\hline
\end{tabular}


TABle 1: Continued.

\begin{tabular}{|c|c|c|c|c|c|c|}
\hline $\begin{array}{l}\text { Herb } \\
\text { name }\end{array}$ & Mol ID & Mol name & Mol structure & Related targets & OB (\%) & $\mathrm{DL}$ \\
\hline & $\begin{array}{l}\text { MOL000374 } \\
\text { MOL000378 }\end{array}$ & $\begin{array}{c}5^{\prime} \text {-Hydroxyiso-muronulatol-2' }{ }^{\prime} 5^{\prime} \\
\text {-di-O-glucoside } \\
\text { 7-O-Methylisomucronulatol }\end{array}$ & & $\begin{array}{l}\text { NOS2, PTGS1, DRD1, CHRM3, F2, } \\
\text { KCNH2, CHRM1, ESR1, AR, } \\
\text { ADRB1, SCN5A, PPARG, F10, } \\
\text { CHRM5, PTGS2, ADRA2C, } \\
\text { CHRM4, RXRA, OPRD1, PDE3A, } \\
\text { ADRA1A, CHRM2, ADRA1B, } \\
\text { SLC6A3, ADRB2, ADRA1D, } \\
\text { SLC6A4, ESR2, DPP4, MAPK14, } \\
\text { GSK3B, HSP90AA1, CHEK1, } \\
\text { PRKACA, PRSS1, CCNA2, NCOA2 }\end{array}$ & 74.69 & 0.30 \\
\hline & MOL000379 & $\begin{array}{l}\text { 9,10-Dimethoxypterocarpan-3-O- } \\
\beta \text {-D-glucoside }\end{array}$ & & PTGS2, TOP2A, NCOA2 & 36.74 & 0.92 \\
\hline & MOL000380 & $\begin{array}{c}\text { (6aR,11aR)-9,10-Dimethoxy- } \\
\text { 6a,11a-dihydro-6H- } \\
\text { benzofurano[3,2-c]chromen-3-ol }\end{array}$ & & $\begin{array}{c}\text { NOS2, PTGS1, CHRM3, F2, } \\
\text { CHRM1, ESR1, SCN5A, PTGS2, } \\
\text { RXRA, ACHE, ADRA1B, ADRB2, } \\
\text { ADRA1D, HSP90AA1, CHRNA7, } \\
\text { PRSS1, NCOA2, NCOA1, CHRM4 }\end{array}$ & 64.26 & 0.42 \\
\hline & MOL000387 & Bifendate & & $\begin{array}{l}\text { PTGS2, KDR, MET, HSP90AA1, } \\
\text { PTGS1, TOP2A }\end{array}$ & 31.10 & 0.67 \\
\hline & MOL000392 & Formononetin & & $\begin{array}{l}\text { NOS2, PTGS1, CHRM1, ESR1, AR, } \\
\text { PPARG, PTGS2, RXRA, PDE3A, } \\
\text { ADRA1A, SLC6A3, ADRB2, } \\
\text { SLC6A4, ESR2, DPP4, MAPK14, } \\
\text { GSK3B, HSP90AA1, MAOB, } \\
\text { CHEK1, PRKACA, PRSS1, CCNA2, } \\
\text { PKIA, F2, ACHE, JUN, PPARG, IL4, } \\
\text { ATP5F1B, ND6, HSD3B2, HSD3B1 }\end{array}$ & 69.67 & 0.21 \\
\hline & MOL000398 & Isoflavanone & & l & 109.99 & 0.30 \\
\hline & MOL000417 & Calycosin & & $\begin{array}{l}\text { NOS2, PTGS1, ESR1, AR, PPARG, } \\
\text { PTGS2, RXRA, PDE3A, ESR2, } \\
\text { DPP4, MAPK14, GSK3B, } \\
\text { HSP90AA1, CHEK1, PRKACA, } \\
\text { PRSS1, CCNA2, NCOA2, ADRB2 }\end{array}$ & 47.75 & 0.24 \\
\hline & MOL000422 & Kaempferol & & $\begin{array}{l}\text { NOS2, PTGS1, AR, PPARG, PTGS2, } \\
\text { HSP90AA1, PRKACA, NCOA2, } \\
\text { DPP4, PRSS1, PGR, F2, CHRM1, } \\
\text { ACHE, SLC6A2, CHRM2, ADRA1B, } \\
\text { TOP2A, F7, RELA, IKBKB, AKT1, } \\
\text { BCL2, BAX, TNFSF15, JUN, } \\
\text { AHSA1, CASP3, MAPK8, MMP1, } \\
\text { STAT1, PPARG, HMOX1, CYP3A4, } \\
\text { CYP1A1, ICAM1, SELE, VCAM1, } \\
\text { NR1I2, CYP1B1, ALOX5, HAS2, } \\
\text { AHR, PSMD3, SLC2A4, NR1I3, } \\
\text { INSR, DIO1, PPP3CA, GSTM1, } \\
\text { GSTM2, AKR1C3, SLPI }\end{array}$ & 41.88 & 0.24 \\
\hline
\end{tabular}


TABle 1: Continued.

\begin{tabular}{|c|c|c|c|c|c|c|}
\hline $\begin{array}{l}\text { Herb } \\
\text { name }\end{array}$ & Mol ID & Mol name & Mol structure & Related targets & OB (\%) & $\mathrm{DL}$ \\
\hline & MOL000433 & FA & & F2, GSK3B & 68.96 & 0.71 \\
\hline & MOL000438 & $\begin{array}{c}\text { (3R)-3-(2-Hydroxy-3,4- } \\
\text { dimethoxyphenyl)chroman-7-ol }\end{array}$ & & I & 67.67 & 0.26 \\
\hline & MOL000439 & $\begin{array}{l}\text { Isomucronulatol-7,2' } \text {-di-O- } \\
\text { glucosiole }\end{array}$ & & TOP2A & 49.28 & 0.62 \\
\hline & MOL000442 & $\begin{array}{l}\text { 1,7-Dihydroxy-3,9-dimethoxy } \\
\text { pterocarpene }\end{array}$ & & PTGS2, RXRA, HSP90AA1, PRSS1 & 39.04 & 0.48 \\
\hline & MOL000098 & Quercetin & & $\begin{array}{l}\text { PTGS1, AR, PPARG, PTGS2, F2, } \\
\text { HSP90AA1, NCOA2, DPP4, } \\
\text { AKR1B1, PRSS1, TOP2A, KCNH2, } \\
\text { SCN5A, F10, ADRB2, MMP3, } \\
\text { PRKACA, F7, RXRA, ACHE, } \\
\text { MAOB, RELA, EGFR, AKT1, } \\
\text { CCND1, BCL2, BCL2L1, FOS, } \\
\text { CDKN1A, EIF6, BAX, CASP9, } \\
\text { PLAU, MMP2, MMP9, MAPK1, } \\
\text { IL10, RB1, TNFSF15, JUN, IL6, } \\
\text { AHSA1, CASP3, TP63, ELK1, } \\
\text { NFKBIA, ODC1, CASP8, TOP1, } \\
\text { RAF1, SOD1, PRKCA, MMP1, } \\
\text { HIF1A, STAT1, RUNX1T1, ERBB2, } \\
\text { PPARG, ACACA, HMOX1, } \\
\text { CYP3A4, CAV1, MYC, F3, GJA1, } \\
\text { CYP1A1, ICAM1, IL1B, SELE, } \\
\text { VCAM1, CXCL8, PRKCB, BIRC5, } \\
\text { DUOX2, NOS3, HSPB1, MGAM, } \\
\text { IL2, NR1I2, CYP1B1, CCNB1, } \\
\text { PLAT, THBD, SERPINE1, IFNG, } \\
\text { ALOX5, IL1A, MPO, TOP2A, } \\
\text { NCF1, ABCG2, HAS2, NFE2L2, } \\
\text { NQO1, PARP1, AHR, PSMD3, } \\
\text { SLC2A4, COL3A1, CXCL11, } \\
\text { CXCL2, DCAF5, NR1I3, CHEK2, } \\
\text { INSR, CLDN4, PPARA, PPARD, } \\
\text { HSF1, CRP, CXCL10, CHUK, SPP1, } \\
\text { RUNX2, RASSF1, E2F1, E2F2, } \\
\text { ACPP, CTSD, IGFBP3, IGF2, } \\
\text { CD40LG, IRF1, ERBB3, PON1, } \\
\text { DIO1, PCOLCE, NPEPPS, HK2, } \\
\text { RASA1, GSTM1, GSTM2 }\end{array}$ & 46.43 & 0.28 \\
\hline
\end{tabular}


ingredients from TCMSP platform were obtained. Additionally, AutoDock tools were also utilized to process the abovementioned protein receptors and ligands, whereas its plugin AutoGrid was used to detect the docking active sites, and a molecular docking process was conducted to obtain the affinity. In our study, the binding energy $\leq-5.0 \mathrm{~kJ} \cdot \mathrm{mol}^{-1}$ was used as the key molecule possessing a better binding affinity with the key targets [33].

2.7. Survival Analysis. In order to better understand the influence of core targets on NSCLC prognosis, a precise analysis of the influence of the ten main targets on the prognosis of all squamous cell carcinomas and adenocarcinomas of NSCLC from TCGA database (https://www.cancer.gov/about-nci/ organization/ccg/research/structural-genomics/tcga) was carried out. The target gene expression was ranked from high to low and used high (50\%) and low (50\%) cutoff values as expression thresholds to divide the high expression group and the low expression group. The resultant worst prognosis of the high expression group relatively reflected that this specific gene might promote tumor development, and vice versa was considered as a protective factor. Data analysis was conducted on $\mathrm{R}$ software, and log-rank test and Kaplan-Meier method were utilized to analyze 1014 samples to obtain survival maps; $p<0.05$ was considered as a statistically significant difference. Moreover, $\mathrm{R}$ software was also used to draw the Sankey diagram in which the width of the extended branch in the figure corresponded to the data flow size, which could be further used to depict the relationship between a certain gene's clinical characteristics, such as smoking, radiation, and racial predilection in NSCLC tumor samples and patient survival, as well as the distribution trend of high and low expressions.

\subsection{Expression of Core Target Genes in NSCLC Tumor Cell} Lines. Since NSCLC has many cell lines, the core genes are distributed differently in various cell lines. For understanding the expression of core genes in the same tumor, we used the Cancer Cell Line Encyclopedia (CCLE) database (https:// sites.broadinstitute. org/ccle) to obtain the cell line gene expression matrix of NSCLC tumors and analyzed the expression of core target genes in different NSCLC tumor cell lines by $\mathrm{R}$ software, to select suitable cell lines for further verification.

\section{Results}

3.1. The Active Ingredients and Chemical Structure of $D B D$. According to the screening conditions of active ingredients, after obtaining 22 active DBD ingredients from the TCMSP database 182 related key targets were acquired. Since the chemical structures of all active ingredients were collected through the TCMSP database, their MOL2 files were also downloaded by the TCMSP database, which is shown as detailed information in Table 1.

3.2. The NSCLC-Related Targets. The NSCLC-related targets were obtained through GeneCards and OMIM database. In the GeneCards database, 5404 related targets of NSCLC were received, while 468 related targets of NSCLC were gained

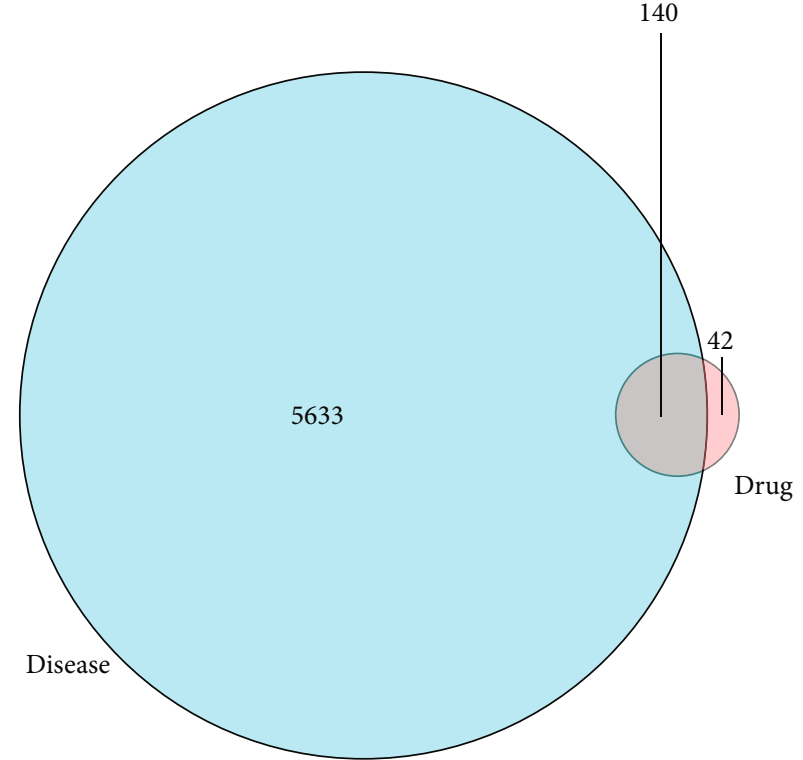

Figure 2: Venn diagram.

from the OMIM database. After the merger of two datasets, deduplication was performed, and 5773 related targets of NSCLC were obtained. Additionally, the intersection of the DBD active ingredient targets Venn diagram and the obtained targets of NSCLC through R software yielded 140 core targets. The results are displayed in Figure 2.

3.3. Protein-Protein Interaction Construction. Several interaction datasets were obtained by incorporating 140 core targets into the string platform and selecting "homo sapiens" to generate the PPI network map, and subsequently, associated protein interaction relationships were acquired. The result is shown in Figure 3. In the PPI network map, there were inherent 140 nodes and 2351 edges in which the nodes represented proteins, edges represented their innate relationships, while the colors from yellow to red represented small to large values.

\subsection{Drug-Ingredient-Target-Disease Network Construction.}

The drug-ingredient-target-disease network diagram was constructed by Cytoscape software, and the results are displayed in Figure 4. The active ingredients and maximum key targets had numerous interactions through computational inference, thus indicating that DBD can treat NSCLC through a precise analysis of multicomponent, multitarget, and multipathway synergistic interactions, which is quite evident from Figure 5. Using Network Analyzer of Cytoscape software, a degree analysis was conducted, and five main DBD ingredients and ten key targets were screened out. The five main active ingredients were quercetin, kaempferol, formononetin, isorhamnetin, and hederagenin, while the ten core targets were HSP90AA1, NCOA2, PPARG, PRKACA, NOS2, PDE3A, PTGS1, PTGS2, ADRB2, and ESR1. Simultaneously, the Cytoscape software was again utilized to investigate the potential collaborative connections between the ten core targets and thereafter demonstrated 


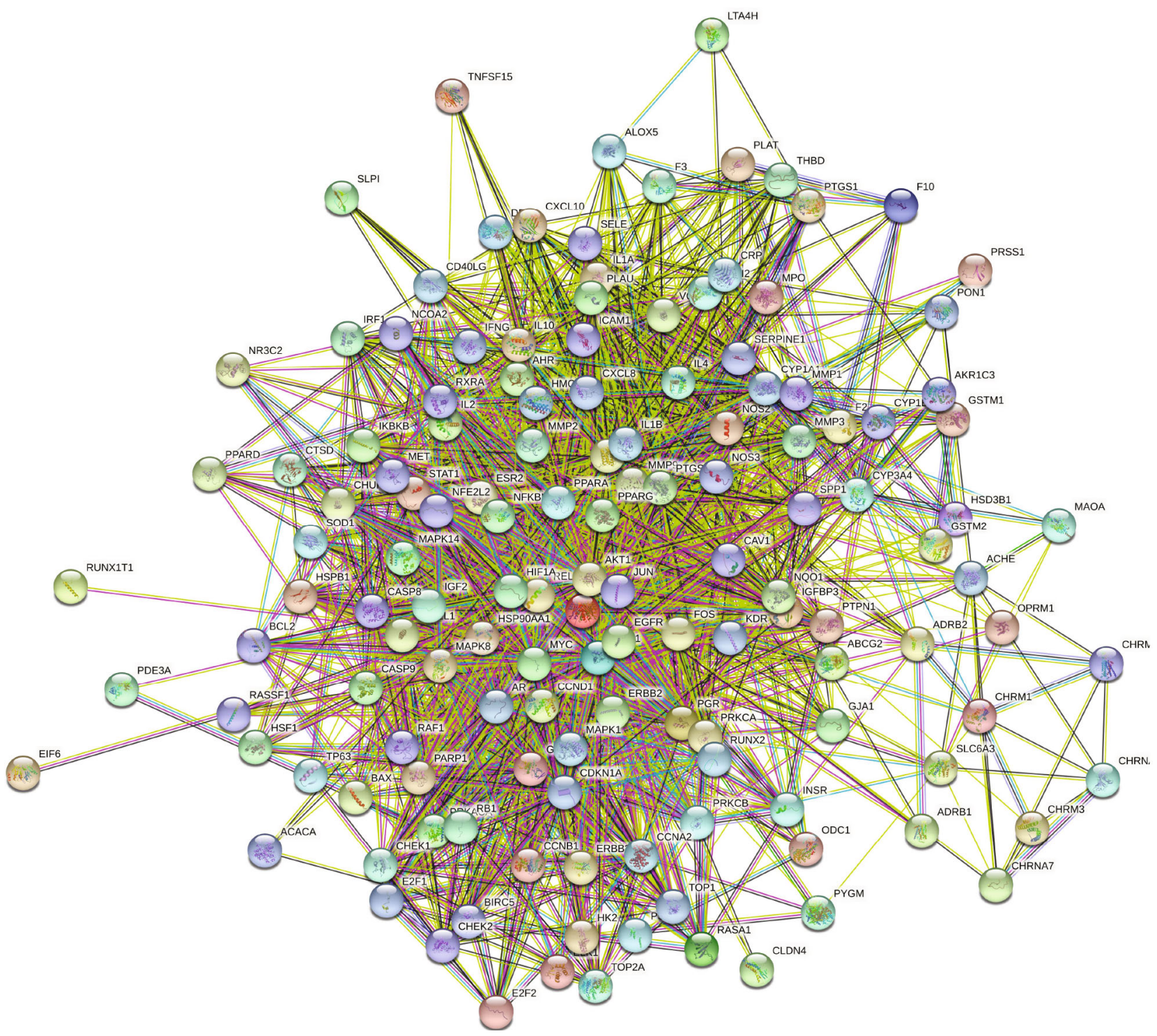

FIgURE 3: PPI network map.

the concept that the redder the color, the closer was its connection with other key targets. The result is shown in Figure 5.

3.5. GO and KEGG Enrichment Analysis. Cytoscape and R software were utilized to perform GO functional enrichment analysis on DBD treatment of NSCLC to explore the possible mechanisms of 140 candidate targets for the treatment of NSCLC, and a total of 131 related biological processes, molecular functions, and cellular components were acquired with a significance value of $p<0.05$. The bubble chart and histogram of the top 20 items are displayed in Figure 6. It was revealed that the coaction targets were mainly enriched in DNA-binding transcription activator activity, RNA polymerase II-specific activity, ubiquitin-like protein ligase binding, endopeptidase activity, protein serine/threonine kinase activity, cytokine receptor binding, ubiquitin-protein ligase binding, etc.
Meanwhile, a similar method was used to perform KEGG enrichment analysis on the DBD treatment regime for NSCLC to explore the possible mechanisms of 140 candidate targets for NSCLC treatment and obtained a total of 174 items with $p<0.05$. The bubble chart and histogram of the top 20 items are shown in Figure 7 . Our study results revealed that the coaction targets were mainly enriched in several diseases: lipid-induced atherosclerosis, hepatitis B, shear stress-induced atherosclerosis, etc., and also participated in different signaling pathways, including the AGERAGE signaling pathway in diabetic complications, IL-17 signaling pathway, and TNF signaling pathway. Figure 8 shows the relevant key targets in both the DBD and AGERAGE signaling pathways in diabetic complications.

3.6. Molecular Docking. The top five target proteins, as well as the top ten active DBD ingredients in the drug-ingredient-target-disease network, were verified by molecular docking to explore the intertwined interactions between the 


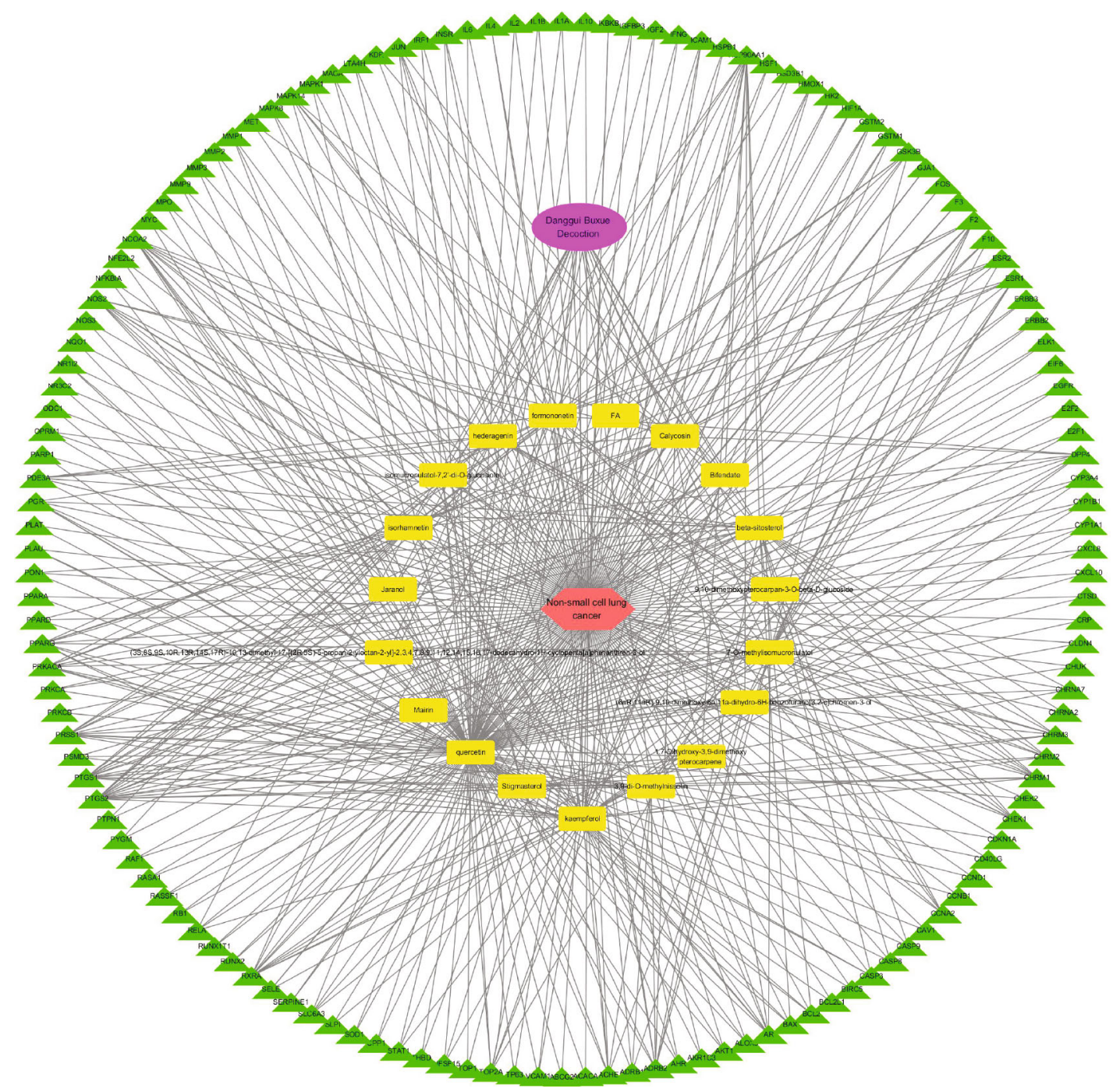

FIGURE 4: Drug-ingredient-target-disease network diagram.

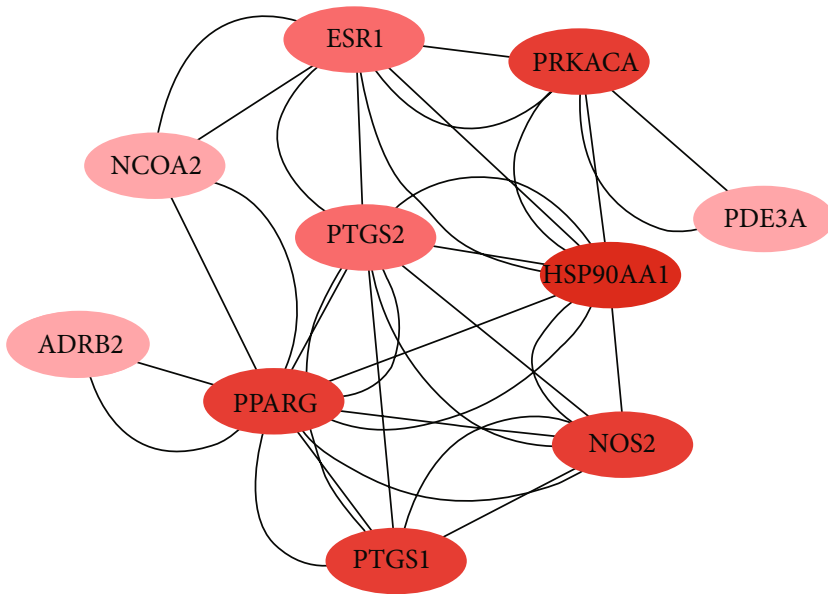

FIgURE 5: Network diagram between ten main targets. receptors and ligands. Moreover, if the binding energy was less than zero, it indicated that the ligand molecules could bind to the receptor protein spontaneously while the affinity less than $-5.0 \mathrm{~kJ} \cdot \mathrm{mol}^{-1}$ revealed that the binding affinity was good. Therefore, it can be easily discerned that the smaller the binding affinity, the greater the molecular docking process. Our study results exhibited that all active ingredients bound well to the target proteins, and every affinity was less than $-5.0 \mathrm{~kJ} \cdot \mathrm{mol}^{-1}$ as depicted in Table 2. In particular, quercetin and hederagenin had a better binding affinity to other target proteins. It was also expressed that quercetin possessed an excellent binding affinity to PTGS1 (affinity $=-9.7 \mathrm{~kJ} \cdot \mathrm{mol}^{-1}$ ) due to the formation of hydrogen bond interactions with the PTGS1 active site, which was the main force promoting its binding capability. Our results also disclosed that these five main active ingredients had better binding affinities towards these protein targets for proper intermolecular interactions.

3.7. Survival Analysis. All squamous cell carcinomas and adenocarcinomas of NSCLC cases were categorized into 


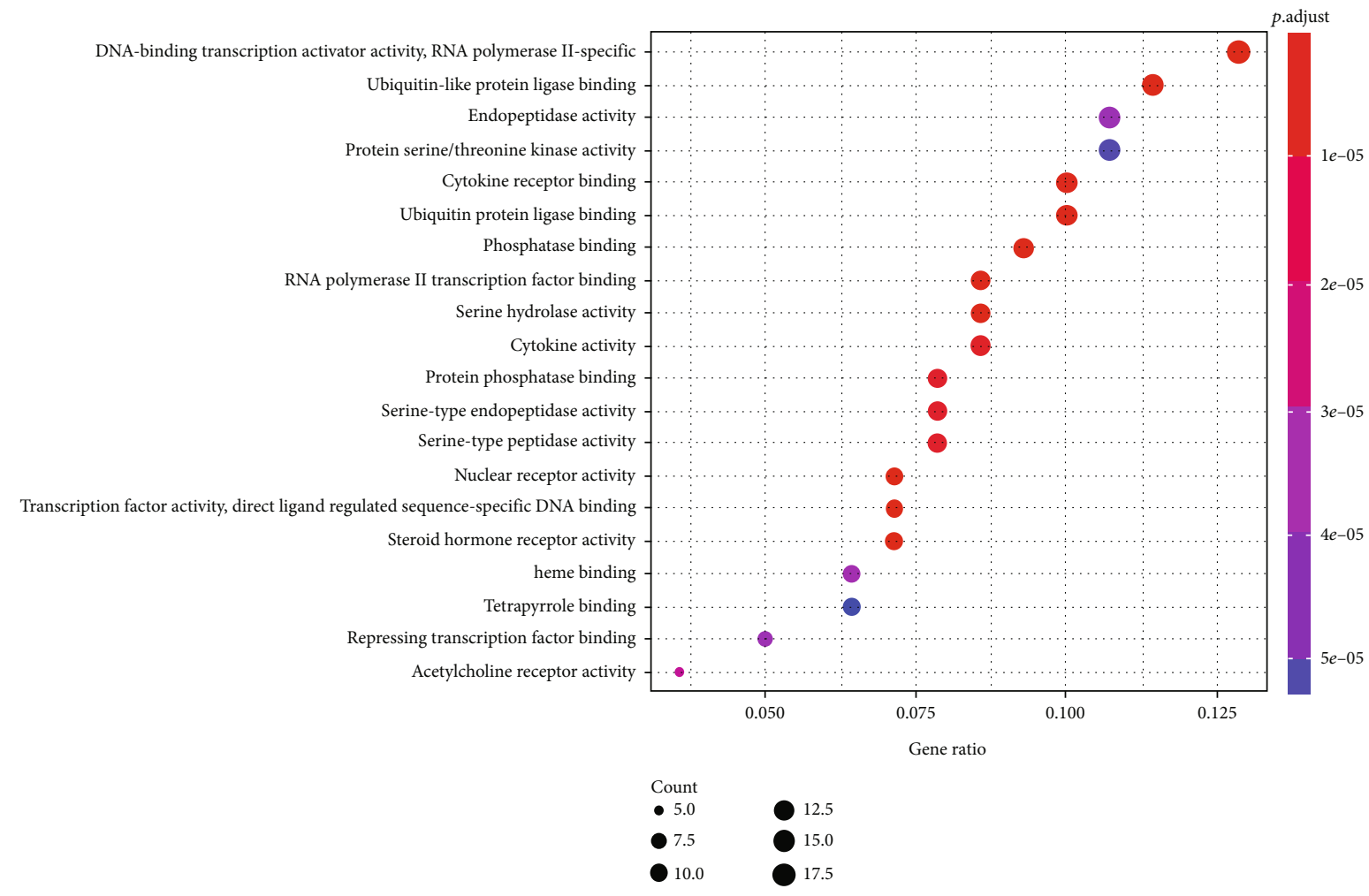

(a)

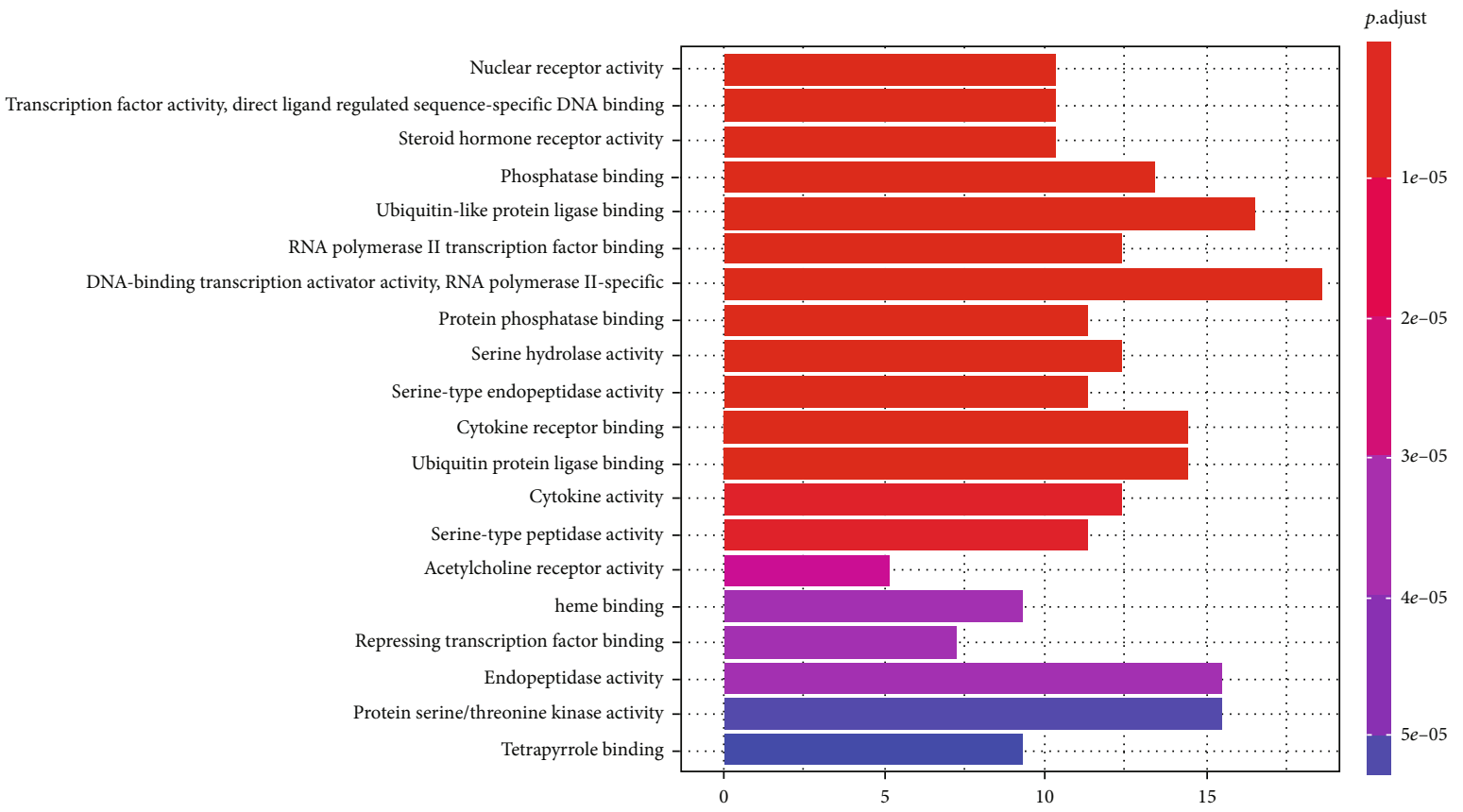

(b)

FIgURE 6: The results of GO functional enrichment analysis. (a) The ordinate includes the biological processes, cell components, and molecular functions involved; the abscissa is the degree of enrichment. The size of the dot represents the number of genes involved; the larger the dot, the greater the number of genes. The lower the $p$ value, the redder the color of the graph, and the higher the enrichment degree. (b) The ordinate is the name of the biological processes, cell components, and molecular functions, and the abscissa is the number of genes enriched in each pathway. The $p$ value indicates the importance of enrichment; the lower the $p$ value, the redder the color of the graph, and the higher the enrichment degree. 


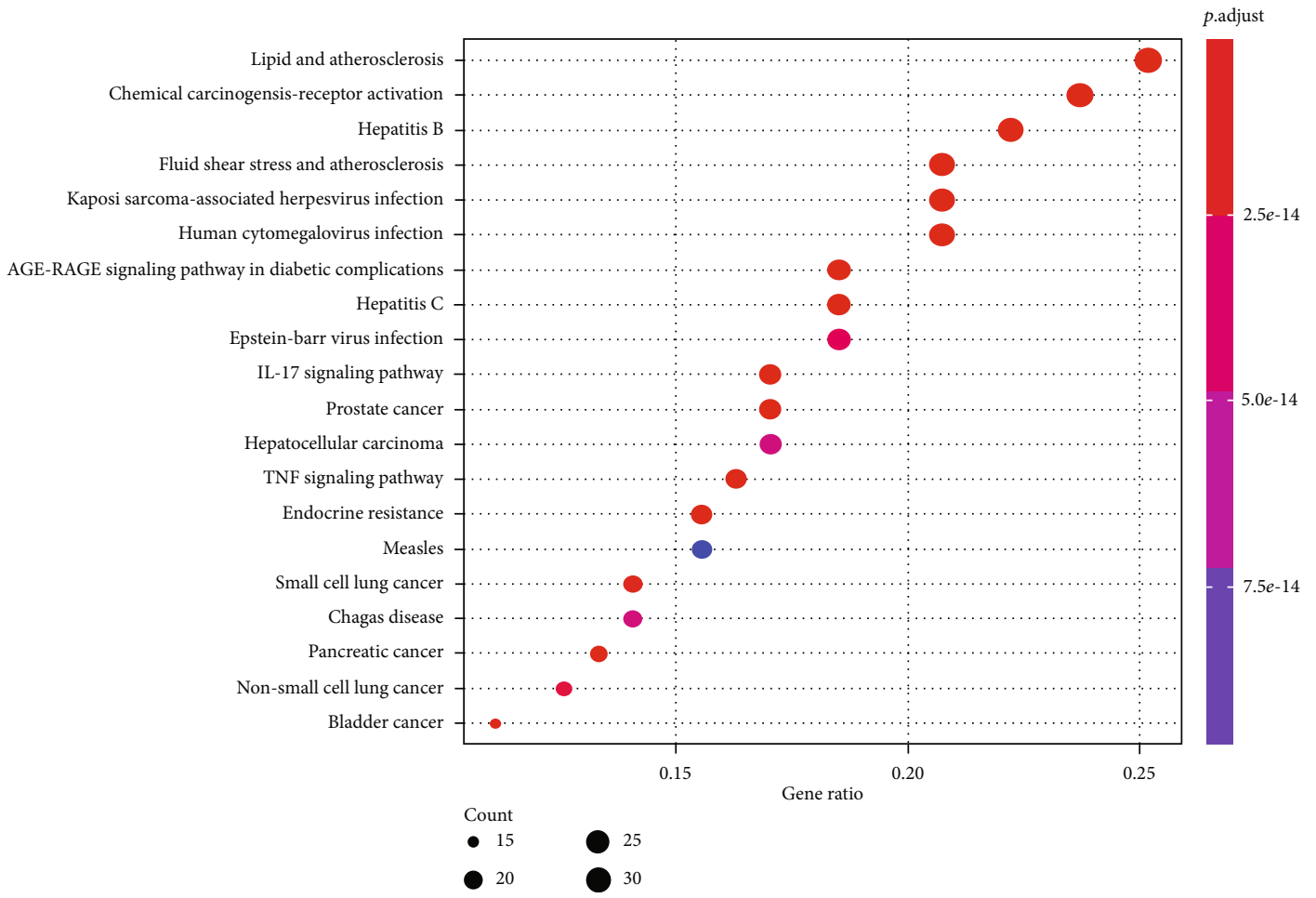

(a)

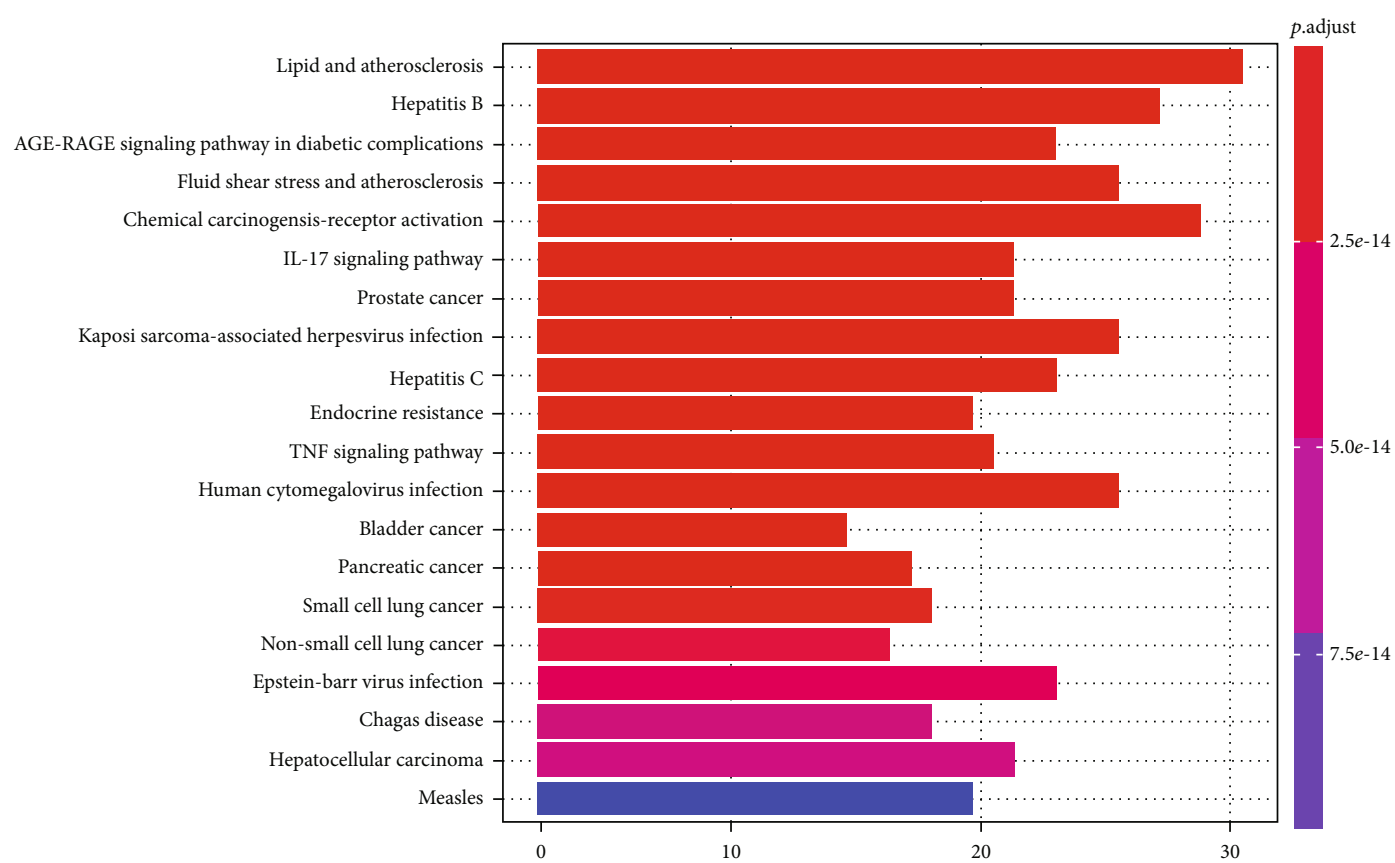

(b)

FIGURE 7: The results of KEGG enrichment analysis. (a) The ordinate includes the related diseases and pathway involved; the abscissa is the degree of enrichment. The size of the dot represents the number of genes involved; the larger the dot, the greater the number of genes. The lower the $p$ value, the redder the color of the graph, and the higher the enrichment degree. (b) The ordinate is the name of the related diseases and pathway, and the abscissa is the number of genes enriched in each pathway. The $p$ value indicates the importance of enrichment; the lower the $p$ value, the redder the color of the graph, and the higher the enrichment degree.

high and low expression groups based on the expression levels of ten core genes, and the correlation between the individual ten core gene's expressions and the prognosis of NSCLC patients was investigated by exploring TCGA data- base. Our study results revealed that the highly expressed genes were associated with poor prognosis, PDE3A was associated with overall survival in all NSCLC patients $(p=0.0384)$, while the overall survival analysis of other nine 


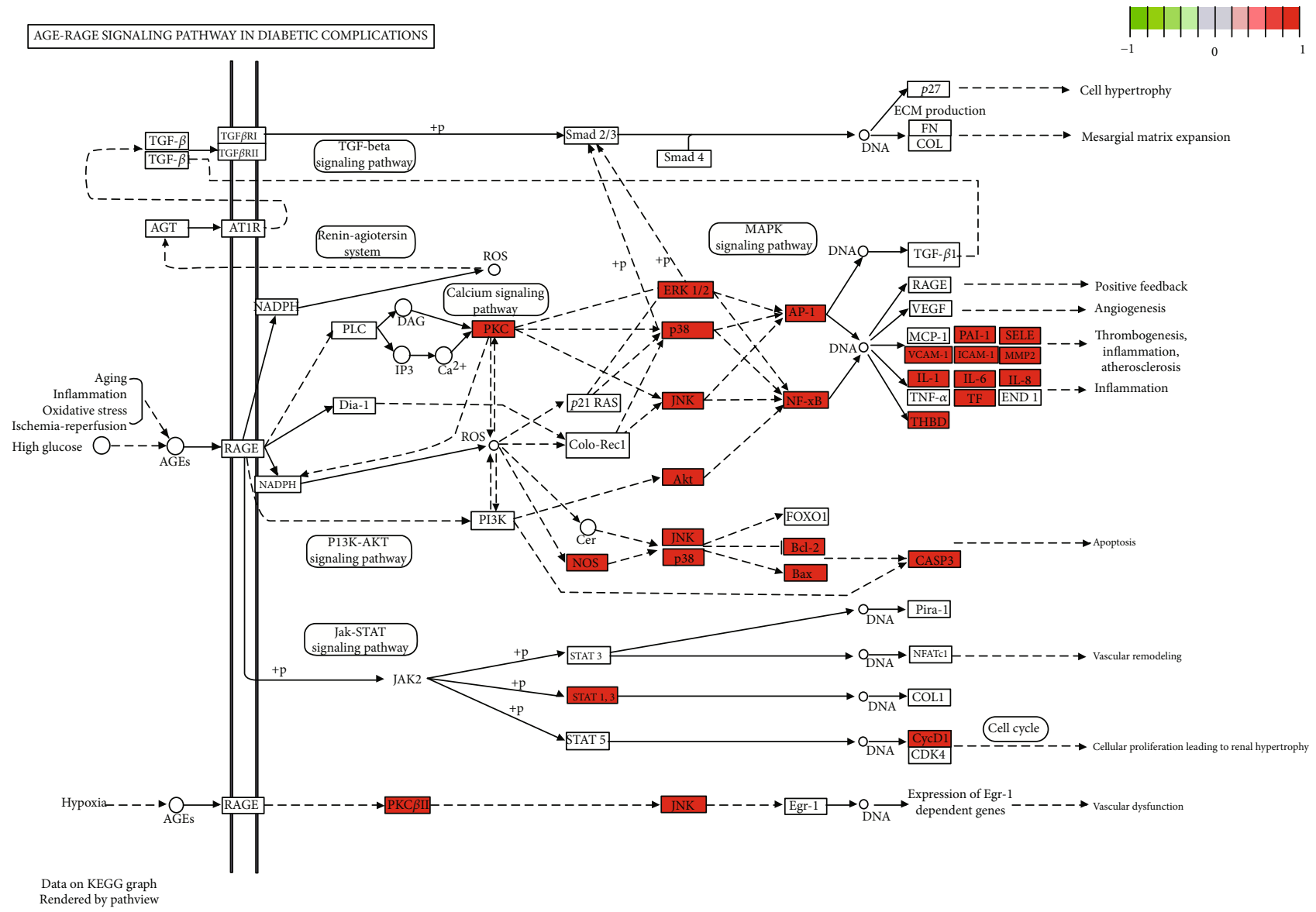

Figure 8: The anti-NSCLC pathway of DBD.

core genes with high and low expression did not have any statistical significance $(p<0.05)$ which is shown in Figure 9. As $p=0.0384$ was utilized to draw the Sankey diagram (Figure 10), the resultant finding unveiled the interwoven relationship between smoking, radiation, racial predilection, the role of PDE3A, and patient survival while showing the potential connection between the width of the extended branch and patient survival.

3.8. Expression of Core Target Genes in NSCLC Tumor Cell Lines. The survival analysis detected that PDE3A had a close connection with patient survival. PDE3A expression in different NSCLC tumor cell lines was analyzed by R software by exploring the CCLE database. Our study results depicted that PDE3A expression was the highest in LU65 and NCIH810, albeit noting that the abscissa represented the PDE3A expression level while the ordinate represented different NSCLC tumor cell lines, as shown in Figure 11.

\section{Discussion}

While lung cancer is still the leading cause of cancer-related deaths globally, non-small-cell lung cancer (NSCLC) is the most common type of lung cancer, accounting for a large proportion of all lung cancer cases [34, 35]. Therefore, choosing the appropriate medicine integrated with standard oncologic care is particularly important for NSCLC patients.
Traditional Chinese medicine is an ancient popular medicine that originated and developed in China. Since it has several unique attributes like the presence of multiple active substances, multiple key targets, and low toxicity [36], it also possesses superior antitumor properties that can be applied in treating different tumors [37]. Our study determined the key active ingredients and possible detailed molecular mechanisms of DBD in the NSCLC treatment through bioinformatics technology to further improve the desired treatment effects as well as to prolong the survival rate of NSCLC patients. In this study, the active ingredients and related targets of DBD were screened from the TCMSP database and further analyzed by GeneCards and OMIM databases to screen out the potential targets for NSCLC. Additionally, the intersection of both DBD ingredient-related targets and the NSCLC-related targets was employed to obtain the core targets. Subsequently, a PPI network and a drug-ingredient-target-disease network were constructed through core targets while using $\mathrm{R}$ software later to perform GO function and KEGG pathway enrichment analysis. The top five active DBD ingredients involved in degree analysis were quercetin, kaempferol, formononetin, isorhamnetin, and hederagenin, while the top ten main core targets included HSP90AA1, NCOA2, PPARG, PRKACA, NOS2, PDE3A, PTGS1, PTGS2, ADRB2, and ESR1. Subsequently, the analysis of five main active ingredients with ten core targets by molecular docking process discovered that the combination of 
TABLE 2: Molecular docking results of 5 main active ingredients and 10 core targets.

\begin{tabular}{|c|c|c|c|c|c|}
\hline Target & PDB ID & Target structure & Active ingredients & Affinity $\left(\mathrm{kJ} \cdot \mathrm{mol}^{-1}\right)$ & Best-docked complex \\
\hline \multirow{5}{*}{ HSP90AA1 } & \multirow{5}{*}{$3 \mathrm{O} 0 \mathrm{I}$} & & Quercetin & -5.6 & \\
\hline & & & Kaempferol & -5.4 & \\
\hline & & & Formononetin & -5.1 & \\
\hline & & & Isorhamnetin & -5.4 & \\
\hline & & & Hederagenin & -5.9 & \\
\hline \multirow{5}{*}{ NCOA2 } & \multirow{5}{*}{$5 \mathrm{EHJ}$} & & Quercetin & -5.5 & \\
\hline & & & Kaempferol & -5.3 & \\
\hline & & & Formononetin & -4.5 & \\
\hline & & & Isorhamnetin & -5.2 & \\
\hline & & & Hederagenin & -5.7 & \\
\hline \multirow{5}{*}{ PPARG } & \multirow{5}{*}{ 6TSG } & & Quercetin & -7.9 & \\
\hline & & & Kaempferol & -7.3 & \\
\hline & & & Formononetin & -6.7 & \\
\hline & & & Isorhamnetin & -7.3 & \\
\hline & & & Hederagenin & -8.2 & \\
\hline \multirow{5}{*}{ PRKACA } & \multirow{5}{*}{ 6ZN0 } & & Quercetin & -7.4 & \\
\hline & & & Kaempferol & -7.1 & \\
\hline & & & Formononetin & -6.4 & \\
\hline & & & Isorhamnetin & -7.6 & \\
\hline & & & Hederagenin & -7.9 & \\
\hline \multirow{5}{*}{ NOS2 } & \multirow{5}{*}{$1 \mathrm{NOS}$} & & Quercetin & -7.4 & \\
\hline & & & Kaempferol & -6.9 & \\
\hline & & & Formononetin & -6.5 & \\
\hline & & & Isorhamnetin & -6.9 & \\
\hline & & & Hederagenin & -6.8 & \\
\hline \multirow{5}{*}{ PDE3A } & \multirow{5}{*}{ 7LRE } & & Quercetin & -8.3 & \\
\hline & & & Kaempferol & -8.4 & \\
\hline & & & Formononetin & -7.5 & \\
\hline & & & Isorhamnetin & -8.4 & \\
\hline & & 5 & Hederagenin & -8.9 & \\
\hline
\end{tabular}


TABLE 2: Continued.

\begin{tabular}{|c|c|c|c|c|c|}
\hline Target & PDB ID & Target structure & Active ingredients & Affinity $\left(\mathrm{kJ} \cdot \mathrm{mol}^{-1}\right)$ & Best-docked complex \\
\hline \multirow{5}{*}{ PTGS1 } & \multirow{5}{*}{$1 \mathrm{EBV}$} & & Quercetin & -9.7 & \\
\hline & & & Kaempferol & -9.6 & \\
\hline & & & Formononetin & -9.3 & \\
\hline & & & Isorhamnetin & -9.5 & \\
\hline & & & Hederagenin & -8.2 & \\
\hline \multirow{5}{*}{ PTGS2 } & \multirow{5}{*}{ 4RUT } & & Quercetin & -7.9 & \\
\hline & & & Kaempferol & -7.7 & \\
\hline & & & Formononetin & -7.7 & \\
\hline & & & Isorhamnetin & -7.8 & \\
\hline & & & Hederagenin & -8.8 & \\
\hline \multirow{5}{*}{ ADRB2 } & \multirow{5}{*}{$3 \mathrm{NY} 9$} & & Quercetin & -7.8 & \\
\hline & & & Kaempferol & -7.8 & \\
\hline & & & Formononetin & -8.1 & \\
\hline & & & Isorhamnetin & -7.8 & \\
\hline & & & Hederagenin & -8.5 & \\
\hline \multirow{5}{*}{ ESR1 } & \multirow{5}{*}{ 6KN5 } & & Quercetin & -6.6 & \\
\hline & & & Kaempferol & -6.7 & \\
\hline & & & Formononetin & -7.0 & \\
\hline & & & Isorhamnetin & -6.6 & \\
\hline & & & Hederagenin & -7.1 & \\
\hline
\end{tabular}

quercetin and PTGS1 had the best binding affinity. Additionally, the analysis of ten main target genes survival and the NSCLC prognosis through TCGA database proved that due to the major distribution of PDE3A in the LU65 and NCI-H810 NSCLC tumor cell lines, it has a statistical significance for the prognostic survival time of NSCLC patients,

According to the drug-ingredient-target-disease network, it was observed that DBD has five main active ingredients: quercetin, kaempferol, formononetin, isorhamnetin, and hederagenin. Since quercetin, a bioflavonoid, can induce Hsp70 inhibition involved in growth inhibition of lung cancer cells, it has a great potential as a chemosensitizer in lung cancer treatment as well as the incorporation of dietary quercetin can also be a promising option for cancer prevention $[38,39]$. Kuo et al., in their study, verified that kaempferol could be used as a radiosensitizer for NSCLC in vitro and in vivo while significantly improving the lethality of tumor cells [40]. Formononetin is a novel herbal isoflavonoid, which when isolated from herbal medicine might act as a potential chemopreventive drug for lung cancer therapy through induction of cell cycle arrest and apoptosis in NSCLC cells [41]. Isorhamnetin, a traditional Chinese medicine used to treat angina pectoris and acute myocardial infarction, displays a series of antitumor activities [42]. Past literary insights on A549 lung cancer cells discovered that isorhamnetin at a concentration of $20 \mu \mathrm{g} / \mathrm{mL}$ can induce apoptosis in A549 cells, upregulate the expression of apoptotic genes Bax, Caspase-3, and p53, and downregulate the expression of $\mathrm{Bcl}-2$, cyclin $\mathrm{D} 1$, and PCNA proteins. Isorhamnetin's mechanism of action may involve apoptosis initiation induced by downregulation of oncogenes and upregulation of apoptotic genes, thus proving that it can significantly inhibit the growth of A549 cells by inducing cell apoptosis [42]. Hederagenin, an oleanolic acid derivative isolated from ivy leaves by displaying potential antitumor activity, might become a promising therapeutic candidate for human colon cancer [43]. A study by Wang et al. reckoned that hederagenin can also induce ROS accumulation 

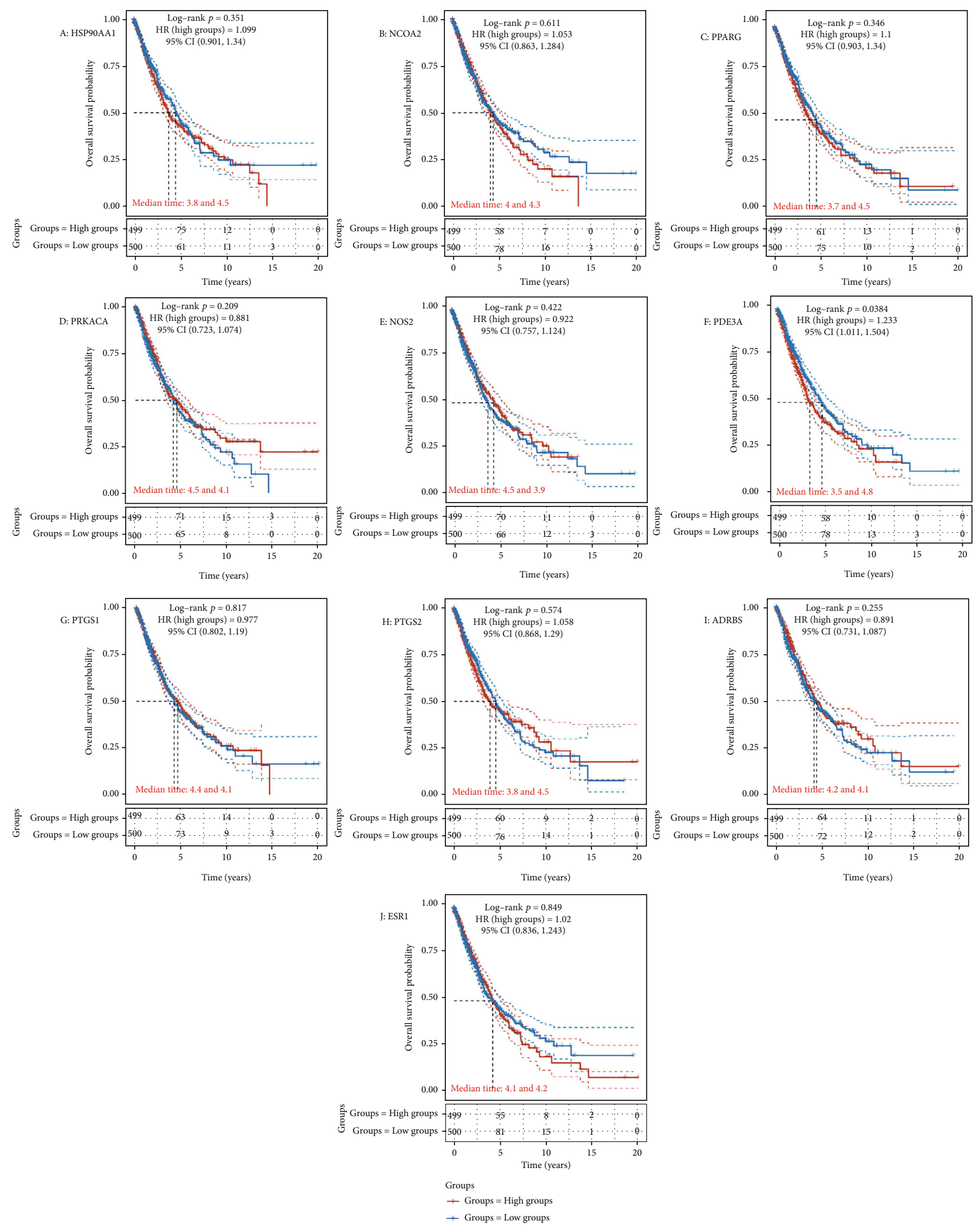

FIGURE 9: Survival analysis for ten main genes by TCGA. 


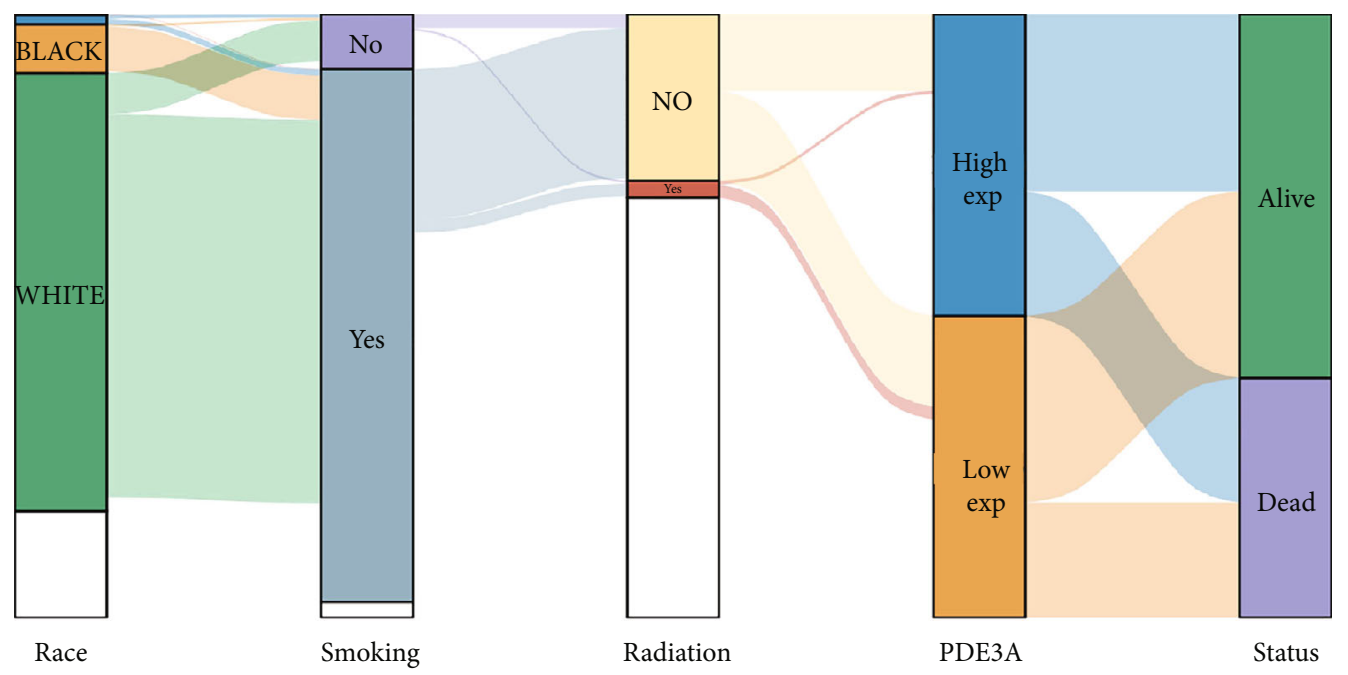

Figure 10: Sankey diagram.

and enhance cisplatin and paclitaxel cytotoxicity in lung cancer cells by blocking autophagic flux [44]. Therefore, hederagenin also has a potential synergistic effect in the treatment of lung cancer.

After exploring 5773 candidate NSCLC targets from GeneCards and OMIM databases, 140 common targets were obtained between the NSCLC and DBD, which were subsequently considered as potential targets for the NSCLC treatment. Through the visual analysis of the drug-ingredient-target-disease network and degree ranking, ten core genes were identified. The degree of association between these genes is shown in Figure 6. These ten core genes included HSP90AA1, NCOA2, PPARG, PRKACA, NOS2, PDE3A, PTGS1, PTGS2, ADRB2, and ESR1and may play an important role in tumor cells, particularly in the process of proliferation, migration, and apoptosis. A previous study disclosed that upregulated KCNQ1OT1 levels in NSCLC tissues and cell lines affirmed that higher KCNQ1OT1 levels were related to the poor progression-free survival of NSCLC patients [45]. Additionally, it was found that the HSP90AA1 expression was reduced after downregulating KCNQ1OT1 levels, which proved that KCNQ1OT1 positively regulated the expression of HSP90AAl by the formation of the miR$27 b-3 p$ sponge. These data revealed the role of KCNQ1OT1 as an oncogene through the modulation of the miR-27b-3p/ HSP90AA1 axis during the progression of NSCLC. Although it was suggested that HSP90AA1 could be a potential target for NSCLC treatment, another protein-coding gene, NCOA2, might be relevant for gastric cancer, liver cancer, or prostate cancer [46-48]. Although many previous studies have hypothesized that NCOA2 might become a potential therapeutic target for NSCLC, further verification is still needed to validate it [49]. PPARG expression may also act as a potential therapeutic agent for NSCLC, especially for lung squamous cell carcinoma (LSCC), since the activation of PPARG expression can inhibit LSCC development and progress by regulating the upstream regulator and downstream marker genes, which are involved in tumor cell proliferation and protein polyubiquitination/ubiquitination [50]. Another study suggested that the loss of NOS2 reduces the growth of lung tumors and the inflammation caused by oncogenic KRAS while stating that KRAS and NOS2 jointly promote the occurrence and inflammation of lung tumors [51]. Moreover, inhibition of NOS2 may have therapeutic value for lung cancer with oncogenic KRAS mutations. It was also evident that highly methylated DNA, downregulated PDE3A in chemoresistant NSCLC cells by forcing PDE3A expression to make A549/Cis cells sensitive to cisplatin. This result indicated that high PDE3A expression might promote the NSCLC treatment by increased efficacy of combination therapies [52]. A network pharmacology study by Wang et al. on NSCLC did not find any effect of PDE3A on NSCLC [53]. Moreover, in our study, the survival analysis of 10 main targets suggested that only PDE3A had statistical significance for the prognosis of NSCLC patients. The survival rate of $\mathrm{PDE} 3 \mathrm{~A}$ in the low expression group was higher than that in the high expression group, further confirming that reducing the expression of PDE3A may improve the quality of life of NSCLC patients. Meanwhile, through the Sankey diagram, it was evident that PDE3A was mainly distributed in the LU65 tumor cell line of NSCLC. Interestingly, the LU65 cell line originated in the Asian populations, especially East Asia, accounting for over $79.97 \%$ of inhabitants [54]. Therefore, PDE3A might be a more suitable potential therapeutic target for Asian NSCLC patients. Wang et al. analyzed 12 human plasma samples by using RNA-Seq and bioinformatics techniques and found seven key targets related to lung tumorigenesis: COX1, COX2, COX3, ND1, ND2, ND4L, and ATP6 [55]. A previous study demonstrated that the SCC pathogenesis caused by COPD is regulated by HSP90AA1, ADRB2, TBL1XR1, and HSPB1. Therefore, these genes can be used as potential therapeutic targets for the treatment of COPDrelated SCC patients [56]. A 2008 study observed that the use of real-time PCR to assess the methylation of the ESR1 promoter in the blood proved very useful for the diagnosis of lung cancer, as these methylated genes might become crucial biomarkers for the early detection of lung cancer. The results also indicated that a comparative evaluation of methylation ratios before and after surgery might be a powerful tool 


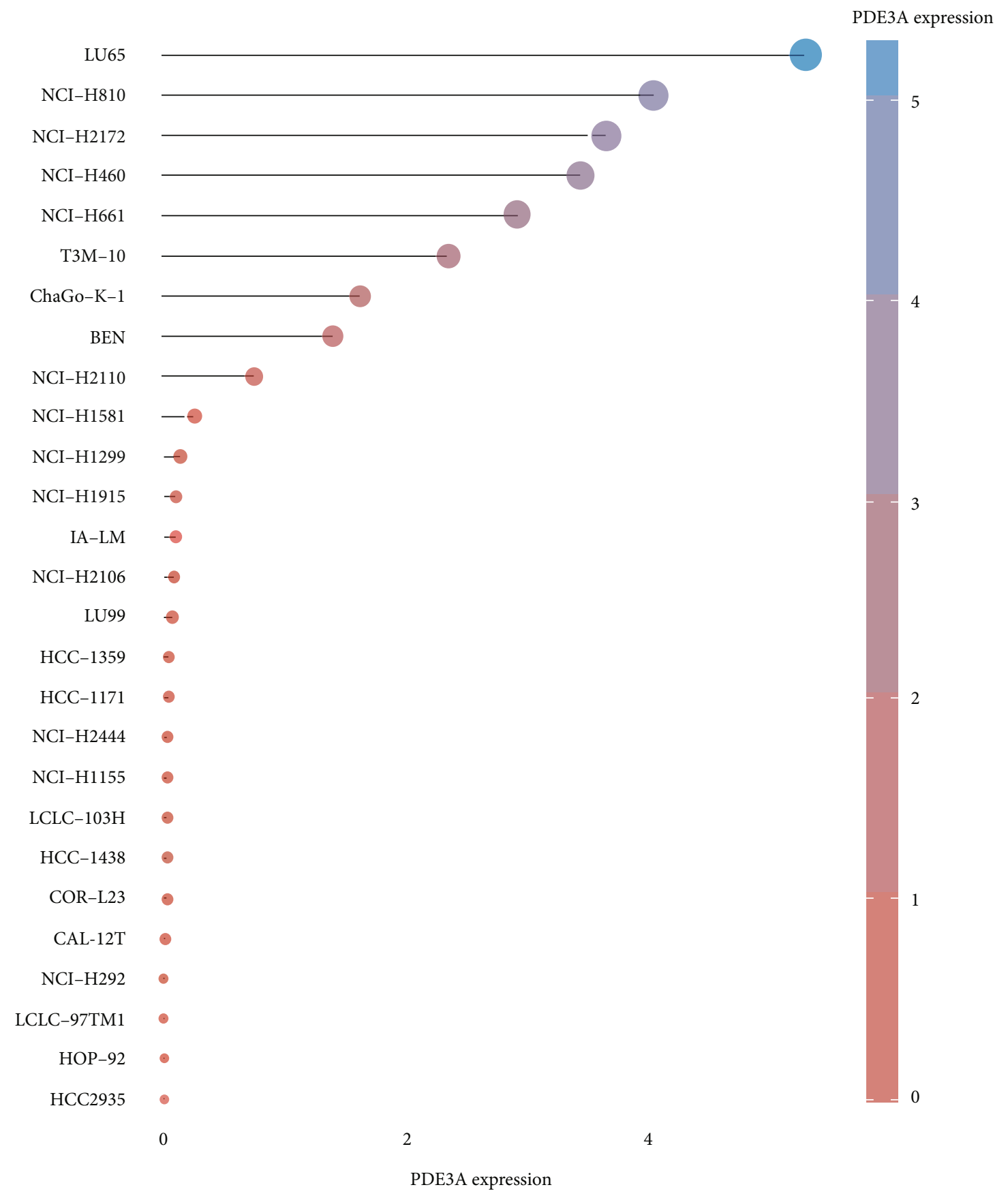

PDE3A expression

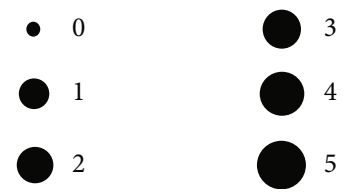

FIgURE 11: Expression of PDE3A in NSCLC tumor cell lines.

for predicting the prognosis of lung cancer patients [57]. Simultaneously, it was suggested that ESR1 mRNA overexpression is innately associated with NSCLC prognosis [58]. Therefore, ESR1 could also become an important key target for treating NSCLC, which is consistent with the results of Wang et al.
[53]. It was also estimated that as PRKACA was a tumor target, it might be helpful as therapy, but due to the lack of evidence, its involvement in the development of lung cancer is debatable.

GO annotation is an important means to examine the function of gene products [59]. Through GO functional 
enrichment analysis, this study inferred all the important biological processes, their cellular compositions, and intermolecular functions involved in the core targets. The details are displayed in Figure 7. In KEGG enrichment analysis, the results were mainly related to the AGE-RAGE signaling pathway in diabetic complications. Accumulation of AGEs and upregulated expression of RAGE is associated with various pathological conditions, including diabetes, cardiovascular diseases, neurodegenerative disorders, and cancer. The role of AGE-RAGE signaling has been previously demonstrated in the progression of various types of cancer and other pathological disorders [60]. Therefore, the regulation of the AGE-RAGE signaling pathway is closely related to tumor development. In the molecular docking process, analysis of five main active ingredients with ten key core targets while calculating the affinity revealed that the affinity of quercetin and PTGS1 was the greatest (affinity $=-9.7 \mathrm{~kJ} \cdot \mathrm{mol}^{-1}$ ), while the binding energy of hederagenin and PDE3A was also good (affinity $=-8.9 \mathrm{~kJ} \cdot \mathrm{mol}^{-1}$ ). These results observed that, although PTGS1 and PDE3A might be the key targets in DBD for treating NSCLC, quercetin and hederagenin may also be effective ingredients for the potential treatment of NSCLC.

However, it should be noted that our study was based on public databases, which had finite information and need to be continuously improved. Additionally, our study also overlooked the influence of concentration, picking time, processing method, and medication time of DBD on NSCLC. But overall, our study is worthy of further exploration and verification to extract the intricate molecular mechanisms governing therapeutic targets for NSCLC treatment.

\section{Conclusions}

Through several bioinformatics methods, our study results predicted the key active ingredients and related targets of DBD as well as explored the potential targets of NSCLC to devise effective drug therapies for the same. Through the enrichment analysis, it was revealed that the potential working mechanism of DBD in NSCLC treatment mainly focused on three crucial interactions: DNA-binding transcription activator activity, RNA polymerase II-specific activity, and chemical carcinogenesis-receptor activation activity. Additionally, few important observations were also discovered. Namely, the AGE-RAGE signaling pathway in diabetic complications, IL17 signaling pathway, and TNF signaling pathway are the three main DBD signaling pathways, and PDE3A and PTGS1 are the potential key genes, while quercetin and hederagenin are the two main active ingredients crucial for NSCLC treatment. Our study results also depicted that DBD can achieve the goal of treating NSCLC while improving the prognosis of NSCLC patients through a synergistic interplay between multiple ingredients, multiple targets, and multiple signaling pathways. Nevertheless, further experimental validation is also required to ascertain effective therapeutic approaches for NSCLC patients. At a later stage, our team might conduct a following pharmacological study on important signaling pathways and targets of DBD against NSCLC and try to provide a functional basis and rational references toward a new future for drug development and targeted therapy.

\author{
Abbreviations \\ CCLE: Cancer Cell Line Encyclopedia \\ DBD: Danggui Buxue Decoction \\ DL: Druglikeness \\ GO: Gene Ontology \\ KEGG: Kyoto Encyclopedia of Genes and Genomes \\ NSCLC: Non-small-cell lung cancer \\ OB: Oral bioavailability \\ OMIM: Online Mendelian Inheritance in Man \\ PPI: $\quad$ Protein-protein interaction \\ TCM: Traditional Chinese medicine \\ TCMSP: Traditional Chinese medicine systems \\ pharmacology \\ TCGA: The Cancer Genome Atlas.
}

\section{Data Availability}

The data used in this research were from open public databases, but the datasets generated and analyzed in this research can be obtained from the corresponding author on request.

\section{Disclosure}

We have modified the current version of this manuscript, which is quite different from the version available in preprint form in Research Square (https://www.researchsquare.com/ article/rs-970748/v1). Although this article was submitted to another journal before, it was rejected but the preprint is still available. We have requested the editor of that journal to remove the preprint. It will be withdrawn soon from Research Square. Therefore, we do not want to affect our progress because of this incident.

\section{Conflicts of Interest}

All authors declare that they have no conflicts of interest.

\section{Authors' Contributions}

BY and HX conceived and designed this study; GYL, MS, and YLL collected the data from the databases; MYY, FYS, $\mathrm{ZYL}$, and BY performed statistical analysis; all authors participated in the drafting of the manuscript and revising it before final submission.

\section{Acknowledgments}

The work was supported by the Science and Technology Project of TCM in Shandong Province (Nos. 2020Z37 and 2020M190).

\section{References}

[1] World Health Organization, Latest global cancer data: cancer burden rises to 19.3 million new cases and 10.0 million cancer deaths in 2020, 2020, https://www.iarc.who.int/news-events/ latest-global-cancer-data-cancer-burden-rises-to-19-3million-new-cases-and-10-0-million-cancer-deaths-in-2020/. 
[2] W. Cao, H. D. Chen, Y. W. Yu, N. Li, and W. Q. Chen, "Changing profiles of cancer burden worldwide and in China: a secondary analysis of the global cancer statistics 2020," Chinese Medical Journal, vol. 134, no. 7, pp. 783-791, 2021.

[3] H. Zeng, W. Chen, R. Zheng et al., "Changing cancer survival in China during 2003-15: a pooled analysis of 17 populationbased cancer registries," The Lancet Global Health, vol. 6, no. 5, pp. e555-e567, 2018.

[4] R. L. Siegel, K. D. Miller, and A. Jemal, "Cancer statistics, 2020," CA: a Cancer Journal for Clinicians, vol. 70, no. 1, pp. 7-30, 2020.

[5] L. Calabrese and X. Mariette, "The evolving role of the rheumatologist in the management of immune-related adverse events (irAEs) caused by cancer immunotherapy," Annals of the Rheumatic Diseases, vol. 77, no. 2, pp. 162-164, 2018.

[6] Z. Ye, Y. Huang, J. Ke, X. Zhu, S. Leng, and H. Luo, "Breakthrough in targeted therapy for non-small cell lung cancer," Biomedicine \& Pharmacotherapy, vol. 133, article 111079, 2021.

[7] Z. H. Caicun, W. A. Jie, B. U. Hong et al., "Chinese experts consensus on immune checkpoint inhibitors for non-small cell lung cancer (2019 version)," Chinese Journal of Lung Cancer, vol. 23 , no. 2 , pp. 65-76, 2020.

[8] Y. T. Lee, Y. J. Tan, and O. CEm, "Molecular targeted therapy: treating cancer with specificity," European Journal of Pharmacology, vol. 834, pp. 188-196, 2018.

[9] J. D. Ransohoff and B. Y. Kwong, "Cutaneous adverse events of targeted therapies for hematolymphoid malignancies," Clinical Lymphoma, Myeloma \& Leukemia, vol. 17, no. 12, pp. 834851, 2017.

[10] Y. Liu, S. Yang, K. Wang et al., "Cellular senescence and cancer: focusing on traditional Chinese medicine and natural products," Cell Proliferation, vol. 53, no. 10, article e12894, 2020.

[11] Z. Yan, Z. Lai, and J. Lin, “Anticancer properties of traditional Chinese medicine," Combinatorial Chemistry \& High Throughput Screening, vol. 20, no. 5, pp. 423-429, 2017.

[12] S. Wang, S. Long, Z. Deng, and W. Wu, "Positive role of Chinese herbal medicine in cancer immune regulation," The American Journal of Chinese Medicine, vol. 48, no. 7, pp. 1577-1592, 2020.

[13] X. L. Su, J. W. Wang, H. Che et al., "Clinical application and mechanism of traditional Chinese medicine in treatment of lung cancer," Chinese Medical Journal, vol. 133, no. 24, pp. 2987-2997, 2020.

[14] P. Wang and Y. Z. Liang, "Chemical composition and inhibitory effect on hepatic fibrosis of Danggui Buxue decoction," Fitoterapia, vol. 81, no. 7, pp. 793-798, 2010.

[15] S. Lan, J. Duan, N. Zeng et al., "Network pharmacology-based screening of the active ingredients and mechanisms of Huangqi against aging," Medicine, vol. 100, no. 17, article e25660, 2021.

[16] J. Fu, Z. Wang, L. Huang et al., "Review of the botanical characteristics, phytochemistry, and pharmacology of Astragalus membranaceus (Huangqi)," Phytotherapy Research, vol. 28, no. 9, pp. 1275-1283, 2014.

[17] X. Q. Shi, S. J. Yue, Y. P. Tang et al., “A network pharmacology approach to investigate the blood enriching mechanism of Danggui buxue decoction," Journal of Ethnopharmacology, vol. 235, pp. 227-242, 2019.

[18] F. Li, R. Tang, L. B. Chen, K. S. Zhang, X. P. Huang, and C. Q. Deng, "Effects of Astragalus combined with Angelica on bone marrow hematopoiesis suppression induced by cyclophosphamide in mice," Biological \& Pharmaceutical Bulletin, vol. 40, no. 5, pp. 598-609, 2017.

[19] Y. D. Li, Y. H. Ma, J. X. Zhao, and X. K. Zhao, "Protection of ultra-filtration extract from Danggui Buxue decoction on oxidative damage in cardiomyocytes of neonatal rats and its mechanism," Chinese Journal of Integrative Medicine, vol. 17, no. 11, pp. 854-859, 2011.

[20] Y. Chen, Y. Y. Tao, and F. H. Li, "Effects of Danggui Buxue decoction on liver fibrosis and hepatic lipid peroxidation in rats," Zhongguo Zhong Xi Yi Jie He Za Zhi, vol. 28, no. 1, pp. 39-42, 2008.

[21] S. H. Feng, B. Zhao, X. Zhan, R. Motanyane, S. M. Wang, and A. Li, "Danggui Buxue decoction in the treatment of metastatic colon cancer: network pharmacology analysis and experimental validation," Drug Design, Development and Therapy, vol. 15, pp. 705-720, 2021.

[22] Q. C. Du, K. Z. Yang, and X. F. Sun, "Efficacy of auxiliary therapy with Danggui Buxue decoction no.1 in treating patients of non-small cell lung cancer at peri-operational stage," Chinese Journal of Integrative Medicine, vol. 15, no. 3, pp. 184-188, 2009.

[23] X. Sun, X. Xu, Y. Chen et al., "Danggui Buxue decoction sensitizes the response of non-small-cell lung cancer to gemcitabine via regulating deoxycytidine kinase and P-glycoprotein," Molecules, vol. 24, no. 10, p. 2011, 2019.

[24] Y. Liu, M. Chang, Z. Hu et al., "Danggui Buxue decoction enhances the anticancer activity of gemcitabine and alleviates gemcitabine-induced myelosuppression," Journal of Ethnopharmacology, vol. 273, article 113965, 2021.

[25] H. Y. Shi, L. Y. Zhang, Y. Z. Tian, and L. L. Shao, "Effect of Jiawei Danggui Buxue decoction on immune function and quality of life in patients with advanced non-small cell lung cancer chemotherapy," Journal of New Chinese Medicine, vol. 49, no. 3, pp. 106-108, 2017.

[26] Y. M. Ma, X. Z. Zhang, Z. Z. Su et al., "Insight into the molecular mechanism of a herbal injection by integrating network pharmacology and in vitro," Journal of Ethnopharmacology, vol. 173, pp. 91-99, 2015.

[27] J. Huang, W. Guo, F. Cheung, H. Y. Tan, N. Wang, and Y. Feng, "Integrating network pharmacology and experimental models to investigate the efficacy of Coptidis and Scutellaria containing Huanglian Jiedu decoction on hepatocellular carcinoma," The American Journal of Chinese Medicine, vol. 48, no. 1, pp. 161-182, 2020.

[28] T. T. Luo, Y. Lu, S. K. Yan, X. Xiao, X. L. Rong, and J. Guo, "Network pharmacology in research of Chinese medicine formula: methodology, application and prospective," Chinese Journal of Integrative Medicine, vol. 26, no. 1, pp. 72-80, 2020.

[29] S. Li, B. Zhang, D. Jiang, Y. Wei, and N. Zhang, "Herb network construction and co-module analysis for uncovering the combination rule of traditional Chinese herbal formulae," $B M C$ Bioinformatics, vol. 11, Supplement 11, p. S6, 2010.

[30] T. Kaur, A. Madgulkar, M. Bhalekar, and K. Asgaonkar, "Molecular docking in formulation and development," Current Drug Discovery Technologies, vol. 16, no. 1, pp. 30-39, 2019.

[31] Y. Wan, L. Xu, Z. Liu et al., "Utilising network pharmacology to explore the underlying mechanism of Wumei Pill in treating pancreatic neoplasms," BMC Complementary and Alternative Medicine, vol. 19, no. 1, p. 158, 2019. 
[32] Q. Ge, L. Chen, M. Tang et al., "Analysis of mulberry leaf components in the treatment of diabetes using network pharmacology," European Journal of Pharmacology, vol. 833, pp. 5062, 2018

[33] X. Ruan, P. Du, K. Zhao et al., "Mechanism of Dayuanyin in the treatment of coronavirus disease 2019 based on network pharmacology and molecular docking," Chinese Medicine, vol. 15, p. 62, 2020.

[34] M. Wang, R. S. Herbst, and C. Boshoff, “Toward personalized treatment approaches for non-small-cell lung cancer," Nature Medicine, vol. 27, no. 8, pp. 1345-1356, 2021.

[35] J. Judd and H. Borghaei, "Combining immunotherapy and chemotherapy for non-small cell lung cancer," Thoracic Surgery Clinics, vol. 30, no. 2, pp. 199-206, 2020.

[36] K. Wang, Q. Chen, Y. Shao et al., "Anticancer activities of TCM and their active components against tumor metastasis," Biomedicine \& Pharmacotherapy, vol. 133, article 111044, 2021.

[37] D. C. Fan, J. Y. Qi, and M. Z. Zhang, "Insights of Chinese medicine on ventricular remodeling: multiple-targets, individualized-treatment," Chinese Journal of Integrative Medicine, vol. 23, no. 9, pp. 643-647, 2017.

[38] S. H. Lee, E. J. Lee, K. H. Min et al., "Quercetin enhances chemosensitivity to gemcitabine in lung cancer cells by inhibiting heat shock protein 70 expression," Clinical Lung Cancer, vol. 16, no. 6, pp. e235-e243, 2015.

[39] A. Murakami, H. Ashida, and J. Terao, "Multitargeted cancer prevention by quercetin," Cancer Letters, vol. 269, no. 2, pp. 315-325, 2008.

[40] W. T. Kuo, Y. C. Tsai, H. C. Wu et al., "Radiosensitization of non-small cell lung cancer by kaempferol," Oncology Reports, vol. 34, no. 5, pp. 2351-2356, 2015.

[41] Y. Yang, Y. Zhao, X. Ai, B. Cheng, and S. Lu, "Formononetin suppresses the proliferation of human non-small cell lung cancer through induction of cell cycle arrest and apoptosis," International Journal of Clinical and Experimental Pathology, vol. 7, no. 12, pp. 8453-8461, 2014.

[42] Q. Li, F. Q. Ren, C. L. Yang et al., "Anti-proliferation effects of isorhamnetin on lung cancer cells in vitro and in vivo," Asian Pacific Journal of Cancer Prevention, vol. 16, no. 7, pp. 30353042, 2015.

[43] B. X. Liu, J. Y. Zhou, Y. Li et al., "Hederagenin from the leaves of ivy (Hedera helix L.) induces apoptosis in human LoVo colon cells through the mitochondrial pathway," BMC Complementary and Alternative Medicine, vol. 14, p. 412, 2014.

[44] K. Wang, X. Liu, Q. Liu et al., "Hederagenin potentiated cisplatin-and paclitaxel-mediated cytotoxicity by impairing autophagy in lung cancer cells," Cell Death \& Disease, vol. 11, no. 8, p. 611, 2020.

[45] Z. Dong, P. Yang, X. Qiu et al., "KCNQ1OT1 facilitates progression of non-small-cell lung carcinoma via modulating miRNA-27b-3p/HSP90AA1 axis," Journal of Cellular Physiology, vol. 234, no. 7, pp. 11304-11314, 2019.

[46] Z. Lin, F. Yang, D. Lu, W. Sun, G. Zhu, and B. Lan, "Knockdown of NCOA2 inhibits the growth and progression of gastric cancer by affecting the Wnt signaling pathway-related protein expression," Technology in Cancer Research \& Treatment, vol. 19, 2020.

[47] K. A. O'Donnell, V. W. Keng, B. York et al., “A Sleeping Beauty mutagenesis screen reveals a tumor suppressor role for Ncoa2/
Src-2 in liver cancer," Proceedings of the National Academy of Sciences of the United States of America, vol. 109, no. 21, pp. E1377-E1386, 2012.

[48] M. P. Silva, J. D. Barros-Silva, and J. Vieira, "NCOA2 is a candidate target gene of 8q gain associated with clinically aggressive prostate cancer," Genes, Chromosomes \& Cancer, vol. 55, no. 4, pp. 365-374, 2016.

[49] J. Liu, Z. He, S. Li, W. Huang, and Z. Ren, "Network pharmacology-based analysis of the effects of Corydalis decumbens (Thunb.) Pers. in non-small cell lung cancer," Evidence-based Complementary and Alternative Medicine, vol. 2021, Article ID 4341517, 12 pages, 2021.

[50] S. Shi, G. Yu, B. Huang, Y. Mi, Y. Kang, and J. P. Simon, "PARG could work as a valid therapeutic strategy for the treatment of lung squamous cell carcinoma," PPAR Research, vol. 2020, Article ID 2510951, 2020.

[51] H. Okayama, M. Saito, N. Oue et al., "NOS2 enhances KRASinduced lung carcinogenesis, inflammation and microRNA-21 expression," International Journal of Cancer, vol. 132, no. 1, pp. 9-18, 2013.

[52] F. M. Tian, C. Y. Zhong, X. N. Wang, and Y. Meng, "PDE3A is hypermethylated in cisplatin resistant non-small cell lung cancer cells and is a modulator of chemotherapy response," European Review for Medical and Pharmacological Sciences, vol. 21, no. 11, pp. 2635-2641, 2017.

[53] Z. Yunwu, W. Yan, W. Yujia et al., "Using network pharmacology and molecular docking to explore the mechanism of Shan $\mathrm{Ci} \mathrm{Gu}$ (Cremastra appendiculata) against non-small cell lung cancer," Frontiers in Chemistry, vol. 9, article 682862, 2021.

[54] J. Dutil, Z. Chen, A. N. Monteiro, J. K. Teer, and S. A. Eschrich, "An interactive resource to probe genetic diversity and estimated ancestry in cancer cell lines," Cancer Research, vol. 79, no. 7, pp. 1263-1273, 2019.

[55] L. Wang, J. Wang, E. Jia, Z. Liu, Q. Ge, and X. Zhao, "Plasma RNA sequencing of extracellular RNAs reveals potential biomarkers for non-small cell lung cancer," Clinical Biochemistry, vol. 83, pp. 65-73, 2020.

[56] L. Wang, H. Zhao, L. Zhang, H. Luo, Q. Chen, and X. Zuo, "HSP90AA1, ADRB2, TBL1XR1 and HSPB1 are chronic obstructive pulmonary disease-related genes that facilitate squamous cell lung cancer progression," Oncology Letters, vol. 19, no. 3, pp. 2115-2122, 2020.

[57] Y. Suga, K. Miyajima, T. Oikawa et al., "Quantitative p16 and ESR1 methylation in the peripheral blood of patients with non-small cell lung cancer," Oncology Reports, vol. 20, no. 5, pp. 1137-1142, 2008.

[58] Y. Teng, Y. Ding, M. Zhang et al., "Genome-wide haplotype association study identifies risk genes for non-small cell lung cancer," Journal of Theoretical Biology, vol. 456, pp. 84-90, 2018.

[59] G. Leale, A. E. Baya, D. H. Milone, P. M. Granitto, and G. Stegmayer, "Inferring unknown biological function by integration of GO annotations and gene expression data," IEEE/ ACM Transactions on Computational Biology and Bioinformatics, vol. 15, no. 1, pp. 168-180, 2018.

[60] B. N. Waghela, F. U. Vaidya, K. Ranjan, A. S. Chhipa, B. S. Tiwari, and C. Pathak, "AGE-RAGE synergy influences programmed cell death signaling to promote cancer," Molecular and Cellular Biochemistry, vol. 476, no. 2, pp. 585-598, 2021. 